\title{
THz-to-Optical Conversion in Wireless Communications Using an Ultra-Broadband Plasmonic Modulator
}

\author{
S. Ummethala ${ }^{1,2, *}$, T. Harter ${ }^{1,2}$, K. Koehnle ${ }^{1,2}, Z$. Li ${ }^{1}$, S. Muehlbrandt ${ }^{1,2}$, Y. Kutuvantavida ${ }^{1,2}$, \\ J. Kemal' ${ }^{1}$, P. Marin-Palomo ${ }^{1}$, J. Schaefer ${ }^{4}$, A. Tessmann ${ }^{5}$, S. K. Garlapati ${ }^{3}$, A. Bacher ${ }^{2}$, \\ L. Hahn ${ }^{2}$, M. Walther ${ }^{5}$, T. Zwick ${ }^{4}$, S. Randel ${ }^{1}$, W. Freude ${ }^{1}$, C. Koos ${ }^{1,2, * *}$ \\ ${ }^{I}$ Institute of Photonics and Quantum Electronics (IPQ), Karlsruhe Institute of Technology (KIT), 76131 Karlsruhe, Germany \\ ${ }^{2}$ Institute of Microstructure Technology (IMT), Karlsruhe Institute of Technology (KIT), 76334, Eggenstein-Leopoldshafen, Germany \\ ${ }^{3}$ Institute of Nanotechnology (INT), Karlsruhe Institute of Technology (KIT), 76334, Eggenstein-Leopoldshafen, Germany \\ ${ }^{4}$ Institute of Radio Frequency Engineering \& Electronics (IHE), Karlsruhe Institute of Technology (KIT), 76131 Karlsruhe, Germany \\ ${ }^{5}$ Fraunhofer Institute for Applied Solid State Physics (IAF), 79108 Freiburg, Germany \\ “sandeep.ummethala@kit.edu, ${ }^{* *}$ christian.koos@kit.edu
}

Future wireless communication networks have to handle data rates of tens or even hundreds of Gbit s ${ }^{-1}$ per link, requiring carrier frequencies in the unallocated terahertz (THz) spectrum ${ }^{1,2}$. In this context, seamless integration of THz links into existing fiber-optic infrastructures ${ }^{3}$ is of great importance to complement the inherent portability and flexibility advantages of wireless networks by the reliable and virtually unlimited capacity of optical transmission systems. On the technological level, this requires novel device and signal processing concepts for direct conversion of data streams between the $\mathrm{THz}$ and the optical domains. Here, we report on the first demonstration of a THz link that is seamlessly integrated into a fiber-optic network using direct terahertz-to-optical (T/O) conversion at the wireless receiver. We exploit an ultra-broadband siliconplasmonic modulator having a $3 \mathrm{~dB}$ bandwidth in excess of $0.36 \mathrm{THz}$ for $\mathrm{T} / \mathrm{O}$ conversion of a $50 \mathrm{Gbit}^{-1}$ data stream that is transmitted on a $0.2885 \mathrm{THz}$ carrier over a $16 \mathrm{~m}$ long wireless link. Optical-to-terahertz $(\mathrm{O} / \mathrm{T})$ conversion at the wireless transmitter relies on photomixing in a unitravelling-carrier photodiode.

Data traffic in wireless communication networks is currently experiencing explosive growth ${ }^{4}$ and will account for more than $60 \%$ of the overall internet traffic by 2021 . To meet the associated capacity challenges, wireless communication networks will have to exploit frequency windows of low atmospheric attenuation in the unallocated $\mathrm{THz}$ spectrum beyond ${ }^{1}$ $0.275 \mathrm{THz}$. Moreover, future wireless links need to be intimately integrated into fiber-optic infrastructures, for example in terahertz-over fiber (ToF) $)^{3}$ or fiber-to-the-antenna (FTTA) ${ }^{5}$ architectures. This calls for seamless connection of optical fibers to $\mathrm{THz}$ transmitter $(\mathrm{Tx})$ and receiver $(\mathrm{Rx})$ front-ends.

At the THz Tx, optoelectronic conversion of data streams from the optical to the THz domain has been demonstrated to offer a variety of advantages ${ }^{1}$ over conventional all-electronic approaches. These advantages include wideband tunability of the carrier frequency and the ability to exploit advanced optical circuitry for generation and multiplexing of data streams prior to conversion to the $\mathrm{THz}$ domain. Wireless transmission with data rates of $100 \mathrm{Gbit} \mathrm{s}^{-1}$ or more have previously been demonstrated $^{6-8}$ by direct optical-to-THz $(\mathrm{O} / \mathrm{T})$ conversion of a wavelength-division multiplexing (WDM) signal in an ultrafast uni-travelling-carrier ${ }^{9}$ (UTC) photodiode. In contrast to that, direct THz-to-optical (T/O) conversion of data signals at the receiver has not yet been shown, and previous transmission experiments $^{6-8,10-12}$ still rely on all-electronic down-conver$\operatorname{sion}^{1}$ of the signals to the baseband using, e.g., sub-harmonic mixers ${ }^{13}$ or Schottky diodes ${ }^{14}$.

In this paper, we report on the first demonstration of a wireless link that is seamlessly integrated into a photonic network, complementing direct $\mathrm{O} / \mathrm{T}$ conversion at the $\mathrm{THz}$ Tx by direct $\mathrm{T} / \mathrm{O}$ conversion at the $\mathrm{THz} \mathrm{Rx}$. The wireless link operates at a carrier frequency of $0.2885 \mathrm{THz}$ with a maximum line rate of $50 \mathrm{Gbit} \mathrm{s}^{-1}$ and bridges a distance of $16 \mathrm{~m}$. The $\mathrm{THz}$ signal is generated by $\mathrm{O} / \mathrm{T}$ conversion in a UTC photodiode. At the receiver, the $\mathrm{THz}$ signal is converted to the optical domain by using an ultra-broadband plasmonic-organic hybrid (POH) modulator. The POH modulator features a flat frequency response $^{15,16}$ up to $0.36 \mathrm{THz}$ along with small footprint of about $600 \mu \mathrm{m}^{2}$, thus lending itself to high-density photonic integration. To the best of our knowledge, this is the first demonstration of direct conversion of a $\mathrm{THz}$ wireless data signal to the optical domain without prior down-conversion to the baseband or to an intermediate frequency. We expect that the combination of direct $\mathrm{O} / \mathrm{T}$ and $\mathrm{T} / \mathrm{O}$ conversion in ultra-compact devices has the potential to greatly accelerate $\mathrm{THz}$ communications and to advance the integration of $\mathrm{THz}$ wireless links into fiberoptic infrastructures. 

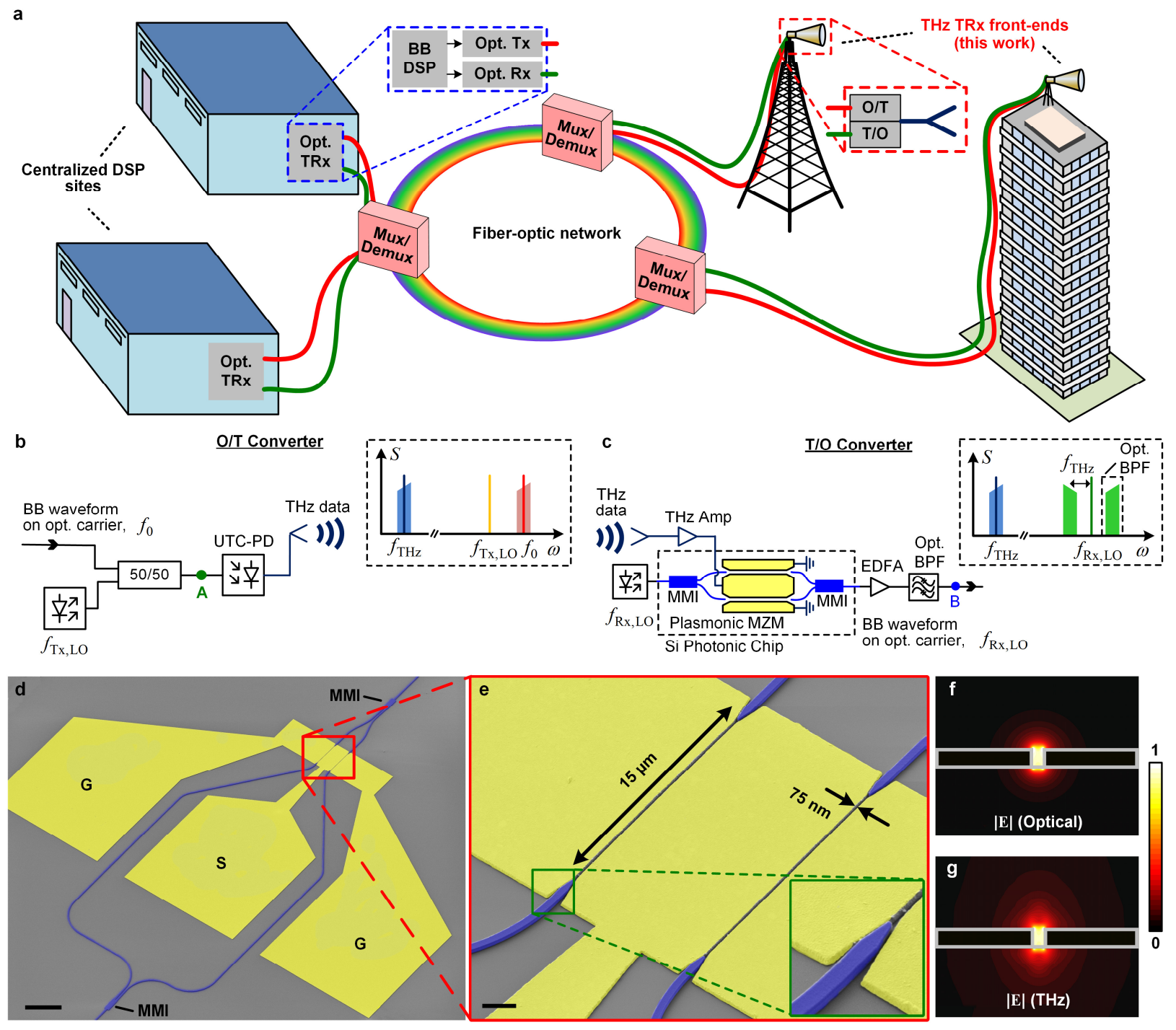

Fig. 1: Seamless integration of THz wireless links into fiber-optic infrastructures by direct optical-to-THz (O/T) and THz-to-optical $(\mathbf{O} / \mathbf{T})$ conversion. a Vision of a future communication network architecture that combines geographically distributed analogue $\mathrm{THz}$ transceiver (TRx) front-ends with powerful centralized digital signal processing (DSP) sites through fiber-optic network infrastructures. Our work focusses on direct $\mathrm{T} / \mathrm{O}$ and $\mathrm{O} / \mathrm{T}$ conversion of analogue waveforms at the $\mathrm{THz}$ front-end, which allows to efficiently interface optical fibers to $\mathrm{THz}$ antennae. This considerably reduces complexity at the antenna site and improves scalability to a large number of THz links or cellular networks. The envisaged concept may thus represent a key step towards overcoming the capacity bottlenecks in wireless communication infrastructures. b Concept of direct $\mathrm{O} / \mathrm{T}$ conversion: The baseband (BB) waveform is first modulated onto an optical carrier at frequency $f_{0}$ and sent to the $\mathrm{O} / \mathrm{T}$ converter. The optical signal is then translated to a $\mathrm{THz}$ carrier by photomixing with a continuous-wave (CW) local oscillator (LO) laser tone at a frequency $f_{\mathrm{Tx}, \mathrm{LO}}$ in an ultra-fast uni-travelling-carrier photodiode (UTC-PD). The THz data signal is radiated into free-space by an antenna. The inset shows the optical data spectrum (center frequency $f_{0}$ ), the LO tone (frequency $f_{\mathrm{Tx}, \mathrm{LO}}$ ), and the $\mathrm{THz}$ spectrum (center frequency $f_{\mathrm{THz}}$ ) after $\mathrm{O} / \mathrm{T}$ conversion. c Concept of direct T/O conversion: The THz data signal is received by an antenna, amplified by a THz amplifier, and then fed to a plasmonic Mach-Zehnder modulator (MZM) for modulation onto a CW carrier at optical frequency $f_{\mathrm{Rx}, \mathrm{LO}}$. After modulation, the optical signal from the MZM contains an upper and a lower modulation sideband. An optical band-pass filter (BPF) is used to suppress the carrier and to select one of the modulation sidebands. The inset depicts an illustration of the spectrum of the THz signal (carrier frequency $f_{\mathrm{THz}}$ ) along with the optical LO tone (frequency $f_{\mathrm{Rx}, \mathrm{LO}}$ ) and the optical signal sidebands generated by THz modulation. The BPF is configured to select one of the sidebands (center frequency $f_{\mathrm{Rx}, \mathrm{LO}}+f_{\mathrm{THz}}$ ). d False-colored scanning electron microscope (SEM) image of a plasmonic MZM with ground-signal-ground (GSG) contact pads (yellow) and integrated silicon photonic waveguides (blue). Scale bar $-20 \mu \mathrm{m}$. e Zoom-in of the plasmonic section of the MZM showing two $15 \mu \mathrm{m}$-long phase modulators with photonic-to-plasmonic mode converters (see Inset). Scale bar $-2 \mu \mathrm{m}$. Each section consists of a plasmonic slot waveguide, featuring a $75 \mathrm{~nm}$-wide gap between two extended gold pads (yellow). f, $\mathbf{g}$ Field profile of the optical quasi-TE mode and the THz field respectively. Both figures indicate the magnitudes of the complex electric field vectors. It can be noticed that the optical and the electronic modes are tightly confined to the slot region, thereby ensuring strong interaction and efficient modulation. 
The concept, presented in Fig. 1a, relies on distributed $\mathrm{THz}$ transceiver (TRx) front-ends that are connected to powerful centralized digital signal processing (DSP) sites through widely deployed fiber-optic network infrastructures that exploit optical carriers to efficiently carry data signals over large distances. The architecture relies on direct $\mathrm{O} / \mathrm{T}$ and $\mathrm{T} / \mathrm{O}$ conversion at the TRx front-end, which is key to efficiently interface optical fibers to $\mathrm{THz}$ antennae. Direct conversion between analogue optical signals and $\mathrm{THz}$ waveforms considerably reduces complexity at the antenna site and improves scalability to a large number of geographically distributed high-performance $\mathrm{THz}$ links or cellular networks. Similarly, the concept of moving computationally expensive digital processing of baseband (BB) signals to centralized sites such as large-scale data centers offers unprecedented network scalability, flexible and efficient sharing of crucial resources as well as improved network resilience. Seamless combination of short-reach $\mathrm{THz}$ links with long-reach fiber-optic networks may thus represent a key step towards overcoming the capacity bottlenecks in wireless communication infrastructures. Note that the scheme presented here is mainly geared towards wireless $\mathrm{THz}$ connections between fixed transmitter and receiver stations rather than towards serving mobile terminals, for which steered $\mathrm{THz}$ beams would be required.

At its heart, the architecture depicted in Fig. 1a relies on $\mathrm{THz}$ transmitter $(\mathrm{Tx})$ and receiver $(\mathrm{Rx})$ front-ends that allow for direct conversion between optical and $\mathrm{THz}$ signals. The underlying concepts are illustrated in Fig. $1 \mathrm{~b}$ and c. After generating the analogue baseband waveform of the data signal at the DSP site, it is modulated onto an optical carrier at frequency $f_{0}$ by an optical transmitter (Opt. Tx) and then sent to the THz Tx through a fiber-optic network. At the THz Tx, see Fig. 1b, the optical signal is then converted to a $\mathrm{THz}$ waveform by photomixing with a continuous-wave $(\mathrm{CW})$ local oscillator (LO) tone at frequency $f_{\mathrm{Tx}, \mathrm{LO}}$ in a UTC photodiode, see Inset of Fig. 1b. The THz data signal, centered at the difference frequency $f_{\mathrm{THz}}=\left|f_{\mathrm{Tx}, \mathrm{LO}}-f_{0}\right|$, is then transmitted into free-space by an antenna. At the T/O converter, Fig. 1c, the THz data signal is received by another antenna and fed to a $\mathrm{THz}$ amplifier. For conversion to an optical carrier, the amplified signal is then coupled to a plasmonic-organic hybrid ${ }^{17-23}$ (POH) MachZehnder modulator (MZM), which is fed by an optical carrier at frequency $f_{\mathrm{Rx}, \mathrm{LO}}$. The POH MZM generates an upper and a lower modulation sideband. An optical band-pass filter (BPF) is used to suppress the carrier and to select one of the modulation sidebands, see Inset of Fig. 1c. This scheme allows for operation over a wide range of $\mathrm{THz}$ frequencies, and does not require any down-conversion to an intermediate frequency before encoding the data onto an optical carrier, and hence considerably reduces the complexity at the THz front-end. After $\mathrm{T} / \mathrm{O}$ conversion, the analogue signal is sent back through fiber-optic network to an optical receiver (Opt. Rx) in the centralized DSP site. The scheme illustrated in Fig. 1c crucially relies on ultra-broadband electro-optic modulators, offering modulation bandwidths that extend into the $\mathrm{THz}$ spectrum. High-speed MZM have previously been demonstrated using lithium niobate as the electro-optic medium - either as bulk material ${ }^{24}$ or as thin films on silicon ${ }^{25}$ or quartz ${ }^{26}$ substrates. However, these devices are usually realized in travelling-wave configuration with typical device lengths on the millimeter or centimeter scale, which is not well suited for high-density integration. Moreover, while some lithium niobate devices show measureable sidebands up to modulation frequencies ${ }^{26}$ of $0.5 \mathrm{THz}$, the underlying $3 \mathrm{~dB}$ bandwidths ${ }^{24-26}$ are so far limited to approximately $0.1 \mathrm{THz}$. These bandwidth limitations can be overcome by plasmonic-organic hybrid modulators ${ }^{17-23}$ that combine organic electro-optic (EO) materials with ultra-compact plasmonic slot waveguides ${ }^{27-29}$. The $\mathrm{POH}$ concept allows to considerably reduce the device footprint and offers a route towards high-density co-integration ${ }^{22}$ with advanced silicon photonic circuitry ${ }^{30,31}$. A fabricated POH MZM is shown in the false-colored scanning electron microscope (SEM) image in Fig. 1d. Light is coupled to the silicon photonic (SiP) chip via on-chip grating couplers (not shown) and propagates in silicon strip waveguides (blue) as a quasi-transverse electric (quasiTE) mode. A multimode interference (MMI) coupler splits the light from the input waveguide and launches it into the two arms of an unbalanced MZM. An MMI coupler at the other end of the MZM combines the modulated signals into an output waveguide which is connected to another grating coupler. Each arm of the MZM contains a $\mathrm{POH}$ phase modulator section comprising a narrow metallic slot (width $w=75 \mathrm{~nm}$ ) between the gold electrodes (yellow), see Fig. 1e. A pair of tapered silicon waveguides in each arm is used to convert ${ }^{32}$ the photonic mode of the silicon strip waveguide to the surface plasmon polariton (SPP) mode in the metallic slot waveguide and vice versa, see Inset of Fig. 1e. The slots are filled with the organic EO material ${ }^{33}$ SEO100. A THz signal applied to the ground-signal-ground (GSG) contacts of the POH MZM leads to a $\mathrm{THz}$ electric field in the slots of each of the two arms and thus creates an optical phase shift. Fig. If and g show that both the optical quasi-TE field and the THz electric field are tightly confined to the metallic slot waveguide, leading to a strong overlap and a high modulation efficiency. The MZM is configured to operate in push-pull mode with phase shifts of equal magnitude but opposite signs in each arm. This is accomplished by an appropriate choice of the poling directions ${ }^{34}$ of 
the EO material with respect to the modulating THz field inside the two slot waveguides. Further details on the device fabrication can be found in the Methods.

Due to the strong interaction of the $\mathrm{THz}$ field and the optical field in the metallic slot waveguide, $\mathrm{POH}$ phase shifters can be very short. This leads to ultra-small parasitic capacitances of the order of a few $\mathrm{fF}$, thereby permitting theoretical RC cutoff frequencies in excess of $1 \mathrm{THz}$ when connected to a signal source with a $50 \Omega$ internal impedance ${ }^{18,34}$. For our experiments, we fabricated a $15 \mu \mathrm{m}$-long POH MZM with a slot width of $75 \mathrm{~nm}$ and characterized its response over an extended frequency range of up to $0.36 \mathrm{THz}$, limited by the signal sources available in our lab. Fig. 2a shows the basic setup for the bandwidth measurement. The optical CW carrier at a frequency $f_{\mathrm{c}}$ is derived from an external-cavity laser (ECL) and launched into the POH MZM that is driven by a small sinusoidal RF or THz signal with varying drive frequency $f_{\mathrm{m}}$. The intensity-modulated optical signal is then amplified by an erbium-doped fiber amplifier (EDFA) and detected by an optical spectrum analyzer to evaluate the phase modulation index $\eta\left(f_{\mathrm{m}}\right)$. Due to the small electrical drive powers of the $\mathrm{THz}$ sources in combination with the comparatively high insertion loss of the specific POH MZM used in our experiments, an EDFA was necessary to boost the optical output power for these measurements. The EDFA could be omitted for later device generations with reduced insertion loss, see Section I of the Supplementary Information for details. For each drive frequency, the optical spectrum exhibits a peak at $f_{\mathrm{c}}$ along with two first-order sidebands at $f_{\mathrm{c}} \pm f_{\mathrm{m}}$. Assuming that the MZM is biased at its quadrature $(3 \mathrm{~dB})$ point and that both arms of the MZM have the same phase modulation index $\eta$, the sideband-to-carrier power ratio $R_{1,0}$ allows to calculate $\eta$ according to the relation ${ }^{35} R_{1,0} \approx \eta^{2} / 4$ for small amplitude of the drive signal $(\eta \ll 1)$, see Methods and Supplementary Section I for more details. Note that the electric drive power $P_{\mathrm{e}}$ provided by our signal source shows a strong dependency on the modulation frequency $f_{\mathrm{m}}$. Since the phase modulation index $\eta$ is proportional to the electric drive voltage $U_{\mathrm{e}}$, we can eliminate the impact of the frequency-dependent drive power $P_{\mathrm{e}}\left(f_{\mathrm{m}}\right)$ by considering the ratio $\eta\left(f_{\mathrm{m}}\right) / U_{\mathrm{e}}\left(f_{\mathrm{m}}\right)$. Normalizing the frequency characteristic of this ratio to its value at a reference modulation frequency of $f_{\mathrm{m}, \mathrm{ref}}=2 \mathrm{GHz}$, we obtain the dimensionless normalized phase modulation index

$$
\eta_{U}\left(f_{\mathrm{m}}\right)=20 \log _{10}\left(\frac{\eta\left(f_{\mathrm{m}}\right) / U_{\mathrm{e}}\left(f_{\mathrm{m}}\right)}{\eta\left(f_{\mathrm{m}, \mathrm{ref}}\right) / U_{\mathrm{e}}\left(f_{\mathrm{m}, \mathrm{ref}}\right)}\right) .
$$

a
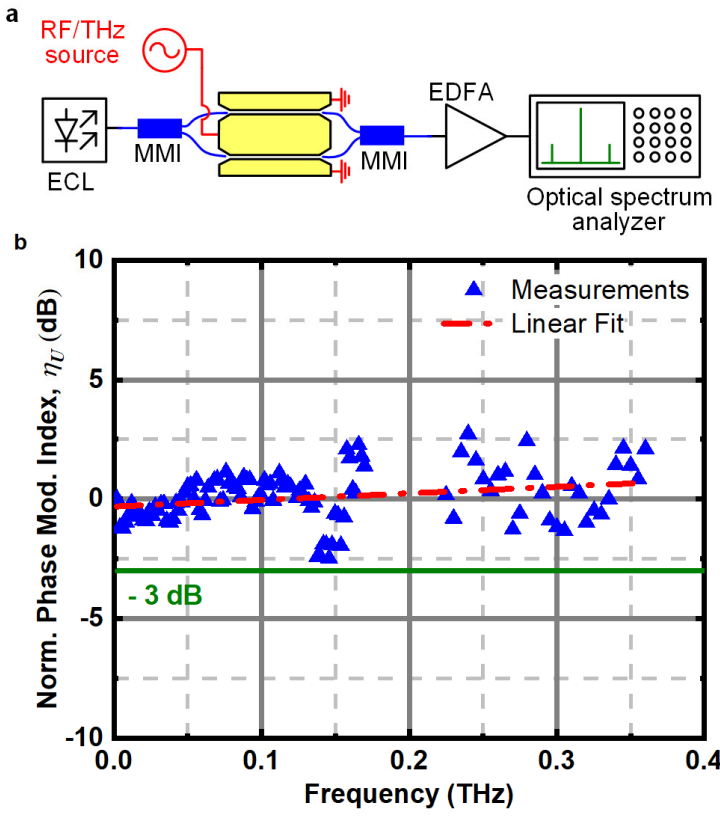

Fig. 2: Bandwidth measurement of $\mathrm{POH}$ modulator. a EXperimental setup for measuring the frequency-dependent phase modulation index $\eta$ of the POH MZM. CW light from an external-cavity laser (ECL) is coupled to the modulator, which is driven by a RF/THz signal. The resulting optical spectrum is recorded by an optical spectrum analyzer and the phase modulation index is extracted from the power ratio of the modulation sidebands and the CW carrier. b Frequency dependence of the normalized modulation index $\eta_{U}$ according to equation (1). The POH MZM features an ultra-broad frequency response up to at least $0.36 \mathrm{THz}$ and shows no indication of a bandwidth limitation. The gap around between $0.17 \mathrm{THz}$ and $0.22 \mathrm{THz}$ is due to the lack of a signal source in this frequency range.

Raw data and details on the evaluation can be found in the Supplementary Section I. The results obtained for our $15 \mu \mathrm{m}$ long POH MZM are shown in Fig. 2b. The device features a flat frequency response over the entire measurement range with no indication of any frequency-dependent decay. The noticeable variations of approximately $\pm 2.5 \mathrm{~dB}$ in the frequency response are attributed to uncertainties of our measurement technique and do not represent an intrinsic property of the POH MZM, see Supplementary Section I for details.

The experimental results hint to a $3 \mathrm{~dB}$ bandwidth that is significantly larger than the highest measured frequency of $0.36 \mathrm{THz}$. To the best of our knowledge, this represents the fastest EO modulator that has so far been published. Our findings are well in line with earlier demonstrations of $\mathrm{POH}$ modulators, where operation up to frequencies of $0.17 \mathrm{THz}$ has been shown ${ }^{23}$. Note that POH devices are not limited to gold as a plasmonic material, but may also be realized by employing CMOS compatible materials ${ }^{36}$, which would allow for co-integration with silicon-based electronics. 
a
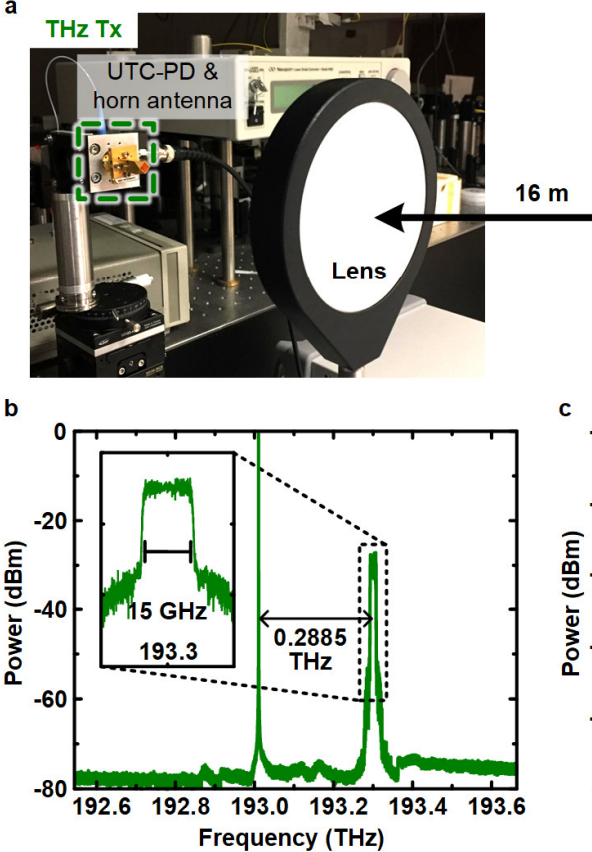
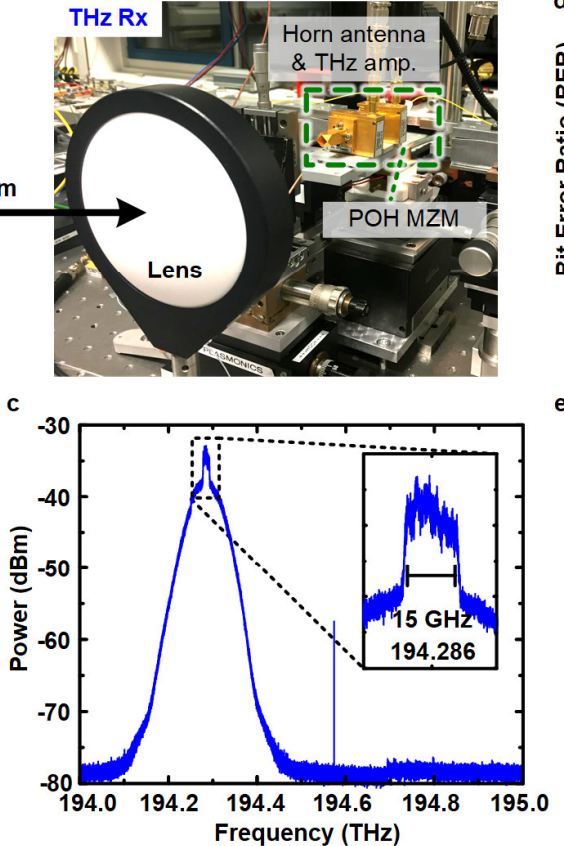

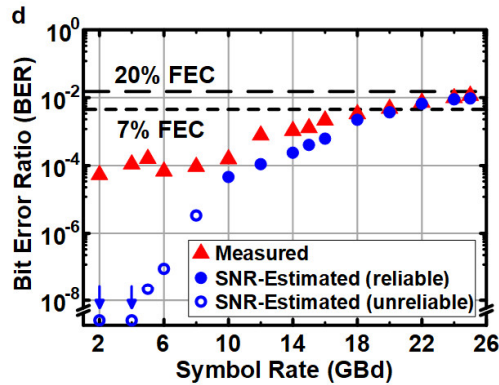

e

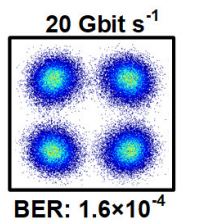

40 Gbit s $^{-1}$

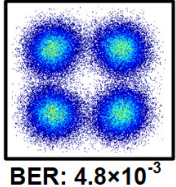

30 Gbit s $^{-1}$

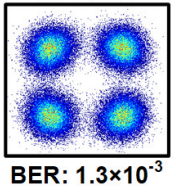

50 Gbit s $^{-1}$

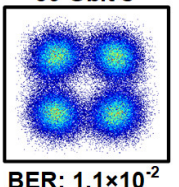

Fig. 3: Demonstration of $\mathbf{T H z}$ wireless data transmission using direct $\mathrm{O} / \mathrm{T}$ and $\mathrm{T} / \mathrm{O}$ conversion. a Photograph of the $\mathrm{THz}$ transmitter (Tx) and receiver (Rx) setup, separated by a distance of $16 \mathrm{~m}$. The THz Tx comprises a high-speed UTC photodiode (UTC-PD), a horn antenna, and a plano-convex polytetrafluoroethylene (PTFE) lens that collimates the THz beam. After travelling over a free-space distance of approximately $16 \mathrm{~m}$, the signal is coupled to the POH MZM through a second lens, a horn antenna, and a two-stage THz amplifier. A more detailed description and a technical sketch of the data transmission setup can be found in Supplementary Section III and in Supplementary Fig. S7. b Optical spectrum measured at position 'A' of the THz Tx shown in Fig. 1b. The spectrum consists of a 15 GBd QPSK data signal (inset) at a carrier frequency of $193.3 \mathrm{THz}$ and a CW tone, detuned from the carrier of the data signal by the targeted THz carrier frequency of $0.2885 \mathrm{THz}$. The THz signal is generated by photomixing in an UTC photodiode. c Filtered optical spectrum measured at position ' $\mathrm{B}$ ' of the THz Rx, see Fig. 1c. Upon modulating the THz signal onto the optical carrier, the upper sideband and the carrier are suppressed by an optical band pass filter such that only the lower sideband and the residual carrier are visible. The lower sideband contains a 15 GBd QPSK signal (inset) centered at $194.286 \mathrm{THz}$. d Bit error ratios (BER) of QPSK signals at different symbol rates upon T/O conversion and coherent intradyne detection. Red triangles represent measured BER obtained from $10^{5}$ symbols of the received signal. Filled blue circles represent the BER estimated from the signal-to-noise power ratio (SNR) in the signal bandwidth. For BER $<10^{-5}$ this estimate becomes increasingly unreliable - the associated BER values are indicated by blue open circles. The measured BER for higher symbol rates $(>8 \mathrm{GBd}$ ) fit well to the estimations. For lower symbol rates, the measured BER differs strongly from the (unreliable) estimations due to phase noise of the LO laser in the coherent optical receiver and due to nonlinear distortions caused by the THz amplifiers, see Section IV of the Supplementary Information for details. The BER stays below the $7 \%$ hard-decision forward error correction (FEC) limit up to a symbol rate of $18 \mathrm{GBd}$. Higher symbol rates require $20 \%$ soft-decision FEC. e Constellation diagrams of the received QPSK signal for data rates of $20 \mathrm{Gbit} \mathrm{s}^{-1}, 30 \mathrm{Gbit} \mathrm{s}^{-1}, 40 \mathrm{Gbit} \mathrm{s}^{-1}$, and $50 \mathrm{Gbit} \mathrm{s}^{-1}$.

For the $\mathrm{THz}$ wireless transmission experiments, we use a second-generation device with slightly longer phase shifter sections $(20 \mu \mathrm{m}$ instead of $15 \mu \mathrm{m})$ featuring a slot width of $75 \mathrm{~nm}$ and the same electro-optic material (SEO100) as the cladding. For this device, we measure an EO figure-of-merit (FoM) of $n_{\mathrm{EO}}^{3} r_{33}=315 \mathrm{pm} / \mathrm{V}$, leading to an estimated $\mathrm{EO}$ coefficient of $r_{33}=64 \mathrm{pm} / \mathrm{V}$. Note that there is vast room to further improve the EO FoM and to hence reduce the drive voltage requirement by using more efficient EO materials. As an example, EO FoM values of $n_{\mathrm{EO}}^{3} r_{33}=1990 \mathrm{pm} / \mathrm{V}$ (calculated for $n_{\mathrm{EO}}=1.83$ and $r_{33}=325 \mathrm{pm} / \mathrm{V}$ ) have been previously demonstrated in $\mathrm{POH}$ devices ${ }^{37}$, and even higher values of $n_{\mathrm{EO}}^{3} r_{33}=2300 \mathrm{pm} / \mathrm{V}\left(r_{33}=390 \mathrm{pm} / \mathrm{V}\right.$ for $\left.n_{\mathrm{EO}}=1.81\right)$ have been recently achieved in silicon-organic hybrid ( $\mathrm{SOH}$ ) modulators $^{38}$. Moreover, the plasmonic losses of the modulator can be greatly reduced by, e.g., using silver for the plasmonic slot waveguide. Together with the highly efficient EO polymers, this may lead to more than a 25 -fold improvement in the $\pi$ voltage-loss product of the POH MZM, see Supplementary Sections V and VI for more details.

Fig. 3a shows photographs of the experimental setups used for the transmission experiment. The THz Tx comprises a highspeed UTC photodiode with a horn antenna to generate the THz signal as well as a plano-convex polytetrafluoroethylene (PTFE) lens that collimates the THz beam. After travelling over a free-space distance of approximately $16 \mathrm{~m}$, the THz signal is coupled to the POH MZM (not shown) through a second 
lens, a horn antenna, and a two-stage $\mathrm{THz}$ amplifier. A more detailed description of the $\mathrm{THz}$ components and a technical sketch of the experimental setup can be found in Supplementary Sections II and III respectively. In the transmission experiment, a quadrature phase-shift keying (QPSK) data stream at a symbol rate of $15 \mathrm{GBd}$ is encoded on an optical carrier at $f_{0}=193.3 \mathrm{THz}$ and superimposed with a CW LO tone at $f_{\text {Tx,LO }}$. Photomixing in the UTC photodiode is used to transfer the optical data stream to a $\mathrm{THz}$ carrier frequency of $f_{\mathrm{Tx}, \mathrm{THz}}=\left|f_{0}-f_{\mathrm{Tx}, \mathrm{LO}}\right|=0.2885 \mathrm{THz}$. Fig. $3 \mathrm{~b}$ shows the optical spectrum at the input of the UTC photodiode, marked as position ' $A$ ' in Fig. $1 b$. At the $\mathrm{THz} \mathrm{Rx}$, the received signal is boosted by two cascaded $\mathrm{THz}$ amplifiers to drive the $\mathrm{POH}$ MZM via a ground-signal-ground (GSG) probe with a hollowcore waveguide. The amplifiers are based on millimeter-wave monolithic integrated circuits (MMIC) and feature a total gain of approximately $40 \mathrm{~dB}$ for frequencies between $0.270 \mathrm{THz}$ and $0.310 \mathrm{THz}$, see Supplementary Section II for details. The POH MZM is biased at its quadrature $(3 \mathrm{~dB})$ point and hence imposes an intensity modulation on the $\mathrm{CW}$ carrier at $f_{\mathrm{Rx}, \mathrm{LO}}=194.57 \mathrm{THz}$ obtained from another ECL. The optical data signal is amplified by erbium-doped fiber amplifiers (EDFA) and sent through an optical filter which suppresses the carrier at $f_{\mathrm{Rx}, \mathrm{LO}}$ along with the upper modulation sideband. The corresponding spectrum taken after the optical filter, i.e., at position 'B' in Fig. 1c, is displayed in Fig. 3c. The optical QPSK signal is detected and evaluated using an optical modulation analyzer with an in-built optical LO for coherent intradyne reception. Note that intensity-modulating a THz QPSK signal onto an optical carrier and selective filtering of one of the sidebands generates an optical QPSK signal even though the THz Rx contains only a simple MZM. Note also that, due to the limited tuning range of the optical filter, we chose to isolate the lower sideband of our optical signal rather than the upper one, as sketched in Fig. 1c.

The QPSK data signal is analyzed offline using standard DSP techniques and the bit error ratio (BER) is extracted. Fig. 3d shows the BER as a function of the QPSK symbol rate, where the red triangles indicate the measured BER, while the filled blue circles correspond to the estimated BER from the signalto-noise power ratio (SNR) of the T/O converted signal. This estimate becomes unreliable for BER $<10^{-5}$ which are represented by blue open circles, see Supplementary Section IV for more details. Up to $18 \mathrm{GBd}$ (line rate $36 \mathrm{Gbit} \mathrm{s}^{-1}$ ), the BER stays below the $7 \%$ hard-decision forward error correction (FEC) limit $^{39}$, while we have to resort to a $20 \%$ soft-decision FEC for higher data rates. For higher symbol rates, the BER agrees well with estimations from the SNR, and is mainly limited by the amplified spontaneous emission (ASE) noise of the first EDFA after the POH MZM (EDFA 1 in Supplementary Fig. S7b). For smaller symbol rates, however, the measured BER differs greatly from the SNR-based (unreliable) estimations due to phase noise of the LO laser in the coherent optical receiver and due to nonlinear distortions caused by the $\mathrm{THz}$ amplifiers, see Supplementary Section IV for a detailed analysis. Fig. 3e shows the QPSK constellations for line rates of $20 \mathrm{Gbit} \mathrm{s}^{-1}$, $30 \mathrm{Gbit} \mathrm{s}^{-1}$, $40 \mathrm{Gbit} \mathrm{s}^{-1}$ and, $50 \mathrm{Gbit} \mathrm{s}^{-1}$ respectively. To the best of our knowledge, this experiment corresponds to the first demonstration of data transmission using direct $\mathrm{THz}$-to-optical conversion.

In summary, we have demonstrated an optical-wireless-optical link with purely optoelectronic frequency conversion both at the THz transmitter and the THz receiver. The link spans a distance of $16 \mathrm{~m}$ and is operated at a carrier frequency of $0.2885 \mathrm{THz}$. Key for this demonstration is a compact ultrabroadband POH modulator offering an ultra-high modulation bandwidth of at least $0.36 \mathrm{THz}$. With a $20 \%$ soft-decision FEC, we achieve a fiber-to-fiber line rate of $50 \mathrm{Gbit} \mathrm{s}^{-1}$. In our current experiments, the transmission distance is limited to $16 \mathrm{~m}$ due to the width of our building. Further device improvements will allow to extend the scheme to transmission distances of $1 \mathrm{~km}$ or more, while still maintaining a reasonable loss margin, see Supplementary Section VI for more details. These results demonstrate the prospects of $\mathrm{POH}$ modulators as powerful subsystems for $\mathrm{THz}$ receiver front-ends. Such devices lend themselves to co-integration with the full portfolio of silicon photonic components that have emerged over recent years ${ }^{30}$, possibly complemented by silicon-based nanoelectronic devices ${ }^{31}$. We believe that direct $\mathrm{T} / \mathrm{O}$ conversion at the $\mathrm{Rx}$ can boost terahertz wireless links to data rates of hundreds of gigabit per second.

\section{Methods}

Fabrication and characterization of plasmonic-organic hybrid modulators: The plasmonic-organic hybrid $(\mathrm{POH})$ modulators used in our experiments are fabricated on standard silicon-on-insulator (SOI) substrates, featuring a $220 \mathrm{~nm}$-thick silicon ( $\mathrm{Si}$ ) device layer and a $2 \mu \mathrm{m}$-thick buried oxide $\left(\mathrm{SiO}_{2}\right)$. Structures are defined by high-resolution electron-beam lithography. A partial Si dry etch step followed by a subsequent full etch of the Si layer is used to form the grating couplers and the $500 \mathrm{~nm}$-wide Si nanowire waveguides. The metallic slots of the plasmonic Mach-Zehnder modulator (MZM) are fabricated via a lift-off process where a $150 \mathrm{~nm}$-thick gold layer is thermally evaporated on a sacrificial layer of poly-methyl methacrylate (PMMA). The metallic slots in the two arms of the MZM are designed to be identical, each featuring a width of $w=75 \mathrm{~nm}$, see Fig. 1e. The Si nanowire waveguide, depicted in blue in Fig. 1e, ends at the plasmonic section of the MZM with a taper tip angle of $12^{\circ}$, see inset of Fig. 1e. These tapers enable efficient conversion between the photonic mode in the silicon waveguide and the surface plasmon polariton (SPP) in the metallic slot with typical conversion losses of only $0.7 \mathrm{~dB}$ per transition ${ }^{17}$. The silicon waveguides in the two arms of the MZM have a geometrical length difference of $80 \mu \mathrm{m}$ to enable adjustment of the MZM operating point by tuning the wavelength. The electro-optic (EO) cladding consists of the commercially available material SEO100, for which 
an EO coefficient of $r_{33}=160 \mathrm{pm} / \mathrm{V}$ has been demonstrated in a thin-film experiment $t^{33}$. The EO material is spin-coated onto the plasmonic MZM and a static electric field is applied across the floating ground electrodes at an elevated temperature for aligning the randomly oriented dipoles of the EO materia $^{34}$. Subsequent cooling preserves the dipole orientation even after removal of the poling field. For a plasmonic slot waveguide length of $L=20 \mu \mathrm{m}$, we measured an insertion loss of $16 \mathrm{~dB}$. The in-device EO figure-of-merit (FoM) and the EO coefficient are estimated to be $n_{\mathrm{EO}}^{3} r_{33}=315 \mathrm{pm} / \mathrm{V}$ and $r_{33}=64 \mathrm{pm} / \mathrm{V}$, respectively, by assuming ${ }^{33} n_{\mathrm{EO}}=1.7$. These values are obtained from the measured $U_{\pi} L$-product of $240 \mathrm{~V} \mu \mathrm{m}$ of the push-pull-operated MZM using the relation $U_{\pi} L=w \lambda_{c} /\left(2 n_{\mathrm{EO}}^{3} r_{33} \Gamma_{\text {slot }}\right)$, Eq. (7) in Ref. ${ }^{34}$ In this equation, the parameters $\lambda_{\mathrm{c}}=1.55 \mu \mathrm{m}$ and $U_{\pi}$ are the operating wavelength, and the measured peak-to-peak modulation voltage that is needed to drive the MZM from constructive to destructive interference at low frequencies. The calculated field interaction factor $\Gamma_{\text {slot }}=0.77$, Eq. (20) in Ref. ${ }^{34}$, describes the interaction between the modulating RF field and the optical field. By measuring on-chip silicon nanowire waveguides of different lengths at an excitation wavelength of $1.55 \mu \mathrm{m}$, we estimate an insertion loss of $6.5 \mathrm{~dB}$ per on-chip grating coupler and a propagation loss of $0.9 \mathrm{~dB} / \mathrm{mm}$ for the silicon waveguide sections.

Bandwidth measurement: The frequency response of the plasmonic modulator is measured by applying a small sinusoidal voltage with variable frequency in the range $2 \mathrm{GHz} \leq f_{\mathrm{m}} \leq 0.36 \mathrm{THz}$. The open-circuit transmissionline electrodes of the POH MZM feature a total length of about $150 \mu \mathrm{m}$, see Supplementary Section I for details. For evaluation of the data, we use a global normalization technique that allows to infer the overall shape of the frequency response despite strong variations in the electrical drive power for various frequency bands. The modulation signal is coupled to the POH MZM by groundsignal-ground (GSG) probes, which have a wave impedance of $Z=50 \Omega$. The open-circuit peak voltage $U_{\mathrm{e}}$ at the terminals of the POH MZM is estimated from the electric drive power $P_{\mathrm{e}}$ which is available at the input of the GSG probe and is measured by replacing the probes by a power meter, see Supplementary Section I for details. To determine the phase modulation index $\eta$, we use a high-resolution optical spectrum analyzer (APEX AP2050) to determine the power of the carrier $f_{\mathrm{c}}$ and of the two first-order intensity modulation sidebands at $f_{\mathrm{c}} \pm f_{\mathrm{m}}$. For larger drive amplitudes, a multitude of modulation sidebands would become visible at frequencies $f_{\mathrm{c}} \pm k f_{\mathrm{m}}(k=1,2,3, \ldots)$. Assuming equal phase modulation indices $\eta$ in both arms, the optical power at each frequency is given by ${ }^{35} P_{\mathrm{o}}\left(f_{\mathrm{c}}+k f_{\mathrm{m}}\right)=P_{\mathrm{i}} J_{\mathrm{k}}^{2}(\eta)\left(1+(-1)^{k} \cos \Delta \phi\right)$, where $P_{\mathrm{i}}$ is the power of the light entering the modulator, $J_{\mathrm{k}}(\eta)$ is the k-th order Bessel function of the first kind, and $\Delta \phi$ is the phase difference between the two interferometer arms of the MZM that determines the operating point. The sideband-to-carrier power ratio of the first-order sidebands at $f_{\mathrm{c}} \pm f_{\mathrm{m}}$ and the spectral line at $f_{\mathrm{c}}$ is hence given by $R_{\mathrm{l}, 0}=P_{\mathrm{o}}\left(f_{\mathrm{c}} \pm f_{\mathrm{m}}\right) / P_{\mathrm{o}}\left(f_{\mathrm{c}}\right)=\alpha J_{1}^{2}(\eta) / J_{0}^{2}(\eta)$ where $\alpha=(1-\cos (\Delta \phi)) /(1+\cos (\Delta \phi))$. For small amplitudes of the modulating $\operatorname{signal}(\eta \ll 1)$, the Bessel functions can be approximated by $J_{0}(\eta)=1$ and $J_{1}(\eta)=\eta / 2$, which leads to $R_{1,0} \approx \alpha \eta^{2} / 4$, see Supplementary Section I. In order to cover the frequency range of interest, we employ various sources: For $2 \mathrm{GHz} \leq f_{\mathrm{m}} \leq 64 \mathrm{GHz}$, the source of an Anritsu 37397C vector network analyzer (VNA) is used. A Keysight VNA (PNA-X, N5247) with different frequency extension modules (OML N5262-60003 and V06VNA2-T/R-A) acts as the RF source in the frequency ranges $70 \mathrm{GHz} \leq f_{\mathrm{m}} \leq 0.11 \mathrm{THz}$ and $0.11 \mathrm{THz} \leq f_{\mathrm{m}} \leq 0.17 \mathrm{THz}$, respectively. The frequency range $0.22 \mathrm{THz} \leq f_{\mathrm{m}} \leq 0.36 \mathrm{THz}$ is covered by an electrical signal that is generated by photomixing of two detuned $\mathrm{CW}$ laser tones in a uni-travelling-carrier (UTC) photodiode. In the range $0.17 \mathrm{THz}<f_{\mathrm{m}}<0.22 \mathrm{THz}$, no source was available in our laboratory. To eliminate the impact of the frequency-dependent drive signal power, the modulation sidebands are measured together with the available electrical power $P_{\mathrm{e}}$ in front of the GSG probes, using a step size of $2 \mathrm{GHz}$ for frequencies up to $0.17 \mathrm{THz}$ and of $5 \mathrm{GHz}$ in the frequencies from $0.22 \mathrm{THz}$ up to $0.36 \mathrm{THz}$. A dedicated calibration procedure is used to ensure global comparability of the modulation indices measured in the various frequency bands, see equation (1) as well as Supplementary Section I for details.
For frequencies up to $65 \mathrm{GHz}$, we use an Anritsu ML2438A power meter, whereas the power at higher frequencies is measured by a waveguide-coupled calorimeter (VDI Erickson PM4).

\section{Data availability}

The data that support the plots within this letter and other findings of this study are available from the corresponding author(s) upon reasonable request.

\section{References}

1. Nagatsuma, T., Ducournau, G. \& Renaud, C. C. Advances in terahertz communications accelerated by photonics. Nat. Photon. 10, 371-379 (2016).

2. Kürner, T. \& Priebe, S. Towards THz communications - Status in research, standardization and regulation. J. Infrared, Millimeter, Terahertz Waves 35, 53-62 (2014).

3. Seeds, A. J., Shams, H., Fice, M. J. \& Renaud, C. C. Terahertz photonics for wireless communications. J. Lightwave Technol. 33, 579-587 (2015).

4. Cisco Visual Networking Index: Forecast and Methodology, 2016-2021. White Paper 22 (2017). Available at:

http://www.cisco.com/c/en/us/solutions/collateral/serviceprovider/visual-networking-index-vni/complete-white-paper-c11481360.pdf.

5. Chow, C. W. et al. $100 \mathrm{GHz}$ ultra-wideband (UWB) fiber-to-the-antenna (FTTA) system for in-building and in- home networks. Opt. Express 18, $11-15$ (2010).

6. Koenig, S. et al. Wireless sub-THz communication system with high data rate. Nat. Photon. 7, 977-981 (2013).

7. Yu, X. et al. $160 \mathrm{Gbit} / \mathrm{s}$ photonics wireless transmission in the $300-500$ GHz band. APL Photonics 1, 081301 (2016).

8. Pang, X. et al. $260 \mathrm{Gbit} / \mathrm{s}$ photonic-wireless link in the $\mathrm{THz}$ band. in IEEE Photonics Conference (IPC) 9-10 (2016).

9. Beling, A. \& Campbell, J. C. InP-based high-speed photodetectors. J. Lightwave Technol. 27, 343-355 (2009).

10. Kanno, A. et al. Coherent terahertz wireless signal transmission using advanced optical fiber communication technology. J. Infrared, Millimeter, Terahertz Waves 36, 180-197 (2015).

11. Nagatsuma, T. et al. Terahertz wireless communications based on photonics technologies. Opt. Express 21, 23736 (2013).

12. Wang, C. et al. 0.34-THz wireless link based on high-order area network applications. IEEE Trans. Terahertz Sci. Technol. 4, 75-85 (2014).

13. Crowe, T. W. GaAs Schottky barrier mixer diodes for the frequency range 1-10 THz. Int. J. Infrared Millimeter Waves 10, 765-777 (1989).

14. Harter, T. et al. $110-\mathrm{m} \mathrm{THz}$ wireless transmission at $100 \mathrm{Gbit} / \mathrm{s}$ using a Kramers-Kronig Schottky barrier diode receiver. in 44th European Conference on Optical Communication (ECOC'18) Th3F.7 (postdeadline paper) (2018)

15. Ummethala, S. et al. Terahertz-to-optical conversion using a plasmonic modulator. in Conference on Lasers and Electro-Optics STu3D.4 (2018).

16. Ummethala, S. et al. Wireless transmission at $0.3 \mathrm{THz}$ using direct THzto-optical conversion at the receiver. in 44th European Conference on Optical Communication (ECOC'18) We4H.3 (2018).

17. Melikyan, A. et al. High-speed plasmonic phase modulators. Nat Photon. 8, 229-233 (2014).

18. Haffner, C. et al. All-plasmonic Mach-Zehnder modulator enabling optical high-speed communication at the microscale. Nat. Photon. 9 , 525-528 (2015).

19. Salamin, Y. et al. Direct conversion of free space millimeter waves to optical domain by plasmonic modulator antenna. Nano Lett. 15, 83428346 (2015).

20. Melikyan, A. et al. Plasmonic-organic hybrid ( $\mathrm{POH})$ modulators for OOK and BPSK signaling at $40 \mathrm{Gbit} / \mathrm{s}$. Opt. Express 23, 9938-9946 (2015).

21. Ayata, M. et al. High-speed plasmonic modulator in a single metal layer. Science 358, 630-632 (2017).

22. Haffner, C. et al. Low-loss plasmon-assisted electro-optic modulator. Nature 556, 483-486 (2018).

23. Hoessbacher, C. et al. Plasmonic modulator with $>170 \mathrm{GHz}$ bandwidth demonstrated at 100 GBd NRZ. Opt. Express 25, 1762-1768 (2017)

24. Macario, J. et al. Full spectrum millimeter-wave modulation. Opt. Express 20, 810-815 (2012).

25. Wang, C. et al. Integrated lithium niobate electro-optic modulators operating at CMOS-compatible voltages. Nature 562, 101-106 (2018). 
26. Andrew J., M. et al. Thin film lithium niobate electro-optic modulator with terahertz operating bandwidth. Opt. Express 26, 14810-14816 (2018).

27. Veronis, G. \& Fan, S. Modes of subwavelength plasmonic slot waveguides. J. Lightwave Technol. 25, 2511-2521 (2007).

28. Veronis, G. \& Fan, S. Guided subwavelength plasmonic mode supported by a slot in a thin metal film. Opt. Lett. 30, 3359-3361 (2005).

29. Pile, D. F. P., Gramotnev, D. K., Oulton, R. F. \& Zhang, X. On longrange plasmonic modes in metallic gaps. Opt. Express 15, 13669 (2007).

30. Urbas, A. M. et al. Roadmap on silicon photonics. J. Opt. 18, 073003 (2016).

31. Atabaki, A. H. et al. Integrating photonics with silicon nanoelectronics for the next generation of systems on a chip. Nature 556, 349-354 (2018).

32. Pile, D. F. P. \& Gramotnev, D. K. Adiabatic and nonadiabatic nanofocusing of plasmons by tapered gap plasmon waveguides. Appl. Phys. Lett. 89, 041111 (2006).

33. Enami, Y., Luo, J. \& Jen, A. K. Y. Short hybrid polymer/sol-gel silica waveguide switches with high in-device electro-optic coefficient based on photostable chromophore. AIP Adv. 1, 042137 (2011).

34. Koos, C. et al. Silicon-organic hybrid ( $\mathrm{SOH})$ and plasmonic-organic hybrid (POH) integration. J. Lightwave Technol. 34, 256-268 (2016).

35. Shi, Y., Yan, L. \& Willner, A. E. High-speed electrooptic modulator characterization using optical spectrum analysis. J. Lightwave Technol. 21, 2358-2367 (2003).

36. Naik, G. V., Shalaev, V. M. \& Boltasseva, A. Alternative plasmonic materials: Beyond gold and silver. Adv. Mater. 25, 3264-3294 (2013).

37. Haffner, C. et al. Harnessing nonlinearities near material absorption resonances for reducing losses in plasmonic modulators. Opt. Mater. Express 7, 2168-2181 (2017).

38. Kieninger, C. et al. Ultra-high electro-optic activity demonstrated in a silicon-organic hybrid (SOH) modulator. Optica 5, 739-748 (2018).

39. Chang, F., Onohara, K. \& Mizuochi, T. Forward error correction for 100 $\mathrm{G}$ transport networks. IEEE Commun. Mag. 48, 48-55 (2010).

\section{Acknowledgements}

This work was supported by the European Research Council (ERC Consolidator Grant 'TeraSHAPE', \# 773248), by the DFG project PACE (\# 403188360) within the Priority Programme "Electronic-Photonic Integrated Systems for Ultrafast Signal Processing" (SPP 2111), by the Alfried Krupp von Bohlen und Halbach Foundation, by the Helmholtz International Research School of Teratronics (HIRST), and by the Karlsruhe Nano Micro Facility (KNMF). We also thank Jingdong Luo and Alex K.-Y. Jen from Soluxra for providing the organic EO material.

This is a post-peer-review, pre-copyedit version of an article published in Nature Photonics. The final authenticated version is available online at: http://dx.doi.org/10.1038/s41566-019-0475-6

\section{Author contributions}

S.U., T.H., W.F. and C.K. developed the concept and designed the experiment. S.U. and Z.L. designed the modulators and fabricated them with support from K.K., S.M., S.K.G., A.B. and L.H. S.U. and J.S. characterized the devices. S.U. and T.H. performed the transmission experiments and analyzed the data together with J.K and P.M.-P. Y.K. developed the poling procedure for the $\mathrm{POH}$ modulators and formulated the organic EO material. A.T. and M.W. developed and provided the $\mathrm{THz}$ MMIC amplifiers. The work was supervised jointly by T.Z., S.R., W.F., and C.K. S.U., W.F., and C.K. wrote the paper. All authors revised the paper.

\section{Conflict of interest}

The authors declare no conflict of interest.

Correspondence and requests for materials should be addressed to S.U. or C.K. 


\section{THz-to-Optical Conversion in Wireless Communications Using an Ultra-Broadband Plasmonic Modulator Supplementary Information}

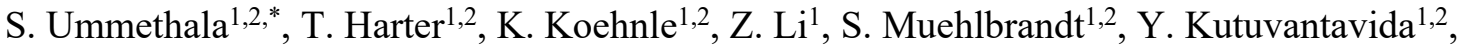
J. Kemal ${ }^{1}$, P. Marin-Palomo ${ }^{1}$, J. Schaefer ${ }^{4}$, A. Tessmann ${ }^{5}$, S. K. Garlapati ${ }^{3}$, A. Bacher ${ }^{2}$, L. Hahn ${ }^{2}$, M. Walther ${ }^{5}$, T. Zwick ${ }^{4}$, S. Randel ${ }^{1}$, W. Freude ${ }^{1}$, C. Koos ${ }^{1,2, * *}$

${ }^{1}$ Institute of Photonics and Quantum Electronics (IPQ), Karlsruhe Institute of Technology (KIT), Karlsruhe, Germany

${ }^{2}$ Institute of Microstructure Technology (IMT), KIT, Eggenstein-Leopoldshafen, Germany

${ }^{3}$ Institute of Nanotechnology (INT), KIT, Eggenstein-Leopoldshafen, Germany

${ }^{4}$ Institute of Radio Frequency Engineering \& Electronics (IHE), KIT, Karlsruhe, Germany

${ }^{5}$ Fraunhofer Institute for Applied Solid State Physics (IAF), Freiburg, Germany

"sandeep.ummethala@kit.edu, ${ }^{* *}$ christian.koos@kit.edu

Contents

I. Frequency response of the plasmonic-organic hybrid (POH) modulator 10

II. Characterization of the uni-travelling-carrier photodiode and the $\mathrm{THz}$ amplifiers .14

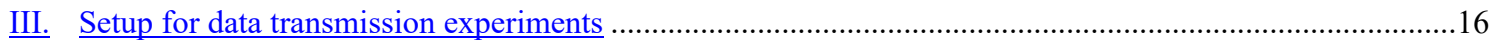

IV. Signal impairment analysis. . .18

V. POH modulator performance and potential for improvements ...............................................................19

VI. General considerations for seamless interfacing of wireless THz links and fiber-optic networks..................20

VII. References .21
} 


\section{Frequency response of the plasmonic-organic hybrid $(\mathrm{POH})$ modulator}

We characterize the frequency response of the plasmonic-organic hybrid (POH) Mach-Zehnder modulator (MZM) by measuring the output spectrum of the intensity-modulated light for different modulation frequencies and by determining the power ratio of the first-order modulation sidebands and the optical carrier. To this end, a continuous-wave (CW) optical carrier at optical frequency $f_{\mathrm{c}}$ (angular frequency $\omega_{\mathrm{c}}=2 \pi f_{\mathrm{c}}$ ) with a power $P_{\mathrm{o}}=18 \mathrm{dBm}$ is launched into the POH MZM, which is driven with a small sinusoidal RF/THz signal at a frequency $f_{\mathrm{m}}$, see Fig. 2a of the main paper. The arm lengths of the POH MZM differ by $27 \mu \mathrm{m}$, and the wavelength of the optical carrier is chosen to operate the device close to its the quadrature point, where the phase shift in the two arms differs by $\Delta \phi=\pi / 2$ in the absence of an electrical modulation signal. The MZM is characterized in the frequency range $2 \mathrm{GHz} \leq f_{\mathrm{m}} \leq 0.36 \mathrm{THz}$. Note that this frequency range is only limited by the equipment available in our lab and not by the speed of the POH MZM itself.

The intensity-modulated signal is detected by an optical spectrum analyzer (OSA, APEX AP2050, 180 MHz resolution bandwidth) which, for small electric drive signals, reveals a peak at the optical carrier frequency $f_{\mathrm{c}}$ and two first-order modulation sidebands at $f_{\mathrm{c}} \pm f_{\mathrm{m}}$. Assuming that the optical signals at the output of the phase-modulating arms of the MZM feature an identical phase modulation index $\eta$, the sideband-to-carrier power ratio $R_{1,0}$ of the first-order sideband and the optical carrier is given by [1]

$$
R_{1,0}=\frac{P_{\mathrm{o}}\left(f_{\mathrm{c}} \pm f_{\mathrm{m}}\right)}{P_{\mathrm{o}}\left(f_{\mathrm{c}}\right)}=\frac{J_{1}^{2}(\eta)(1-\cos \Delta \phi)}{J_{0}^{2}(\eta)(1+\cos \Delta \phi)}
$$

In this relation, $\Delta \phi$ denotes difference of the phase shifts in both arms in the absence of a modulating electrical signal and hence defines the operating point of the device, and $J_{0}$ and $J_{1}$ are the $0^{\text {th }}$ and the $1^{\text {st }}$-order Bessel functions of the first kind. For small electric drive amplitudes $U_{\mathrm{e}}$ of the modulating signal $(\eta \ll 1)$, the Bessel functions can be approximated by $J_{0}(\eta)=1$ and $J_{1}(\eta)=\eta / 2$ which leads to

$$
R_{1,0}=\alpha \frac{\eta^{2}}{4}, \quad \alpha=\frac{(1-\cos \Delta \phi)}{(1+\cos \Delta \phi)}
$$

If the modulator is operated exactly at its quadrature point $\Delta \phi=\pi / 2$, then the correction factor $\alpha=1$. Exact adjustment of the operating point during the experiment, however, turned out to be difficult due to noise and distortions of the $\cos ^{2}$-type spectral power transfer function of the unbalanced POH MZM. To reduce the associated uncertainties, we record the power transmission spectrum of the device over an extended spectral range to estimate the exact phase shift $\Delta \phi$ and hence consider the associated correction factor $\alpha$ for the data evaluation. To this end, we fit the measured power transmission spectrum with a model function. We assume that after eliminating the impact of the grating couplers, the power transfer function of the POH MZM is essentially given by $T(\omega) \propto \cos ^{2}(\Delta \phi / 2)$ with $\Delta \phi=\beta(\omega) \Delta L$, where $\beta(\omega)$ is the frequency-dependent propagation constant and $\Delta L$ is the path length difference between the two arms of the MZM. For fitting the measured data, we additionally allow for a nonperfect extinction ratio of the MZM, and we adopt a second-order Taylor series expansion of $\beta(\omega)$ about the center frequency $\omega_{0}$ of the tuning range for taking into account chromatic dispersion. This leads to a model function of the form

$$
T(\omega)=\mathrm{A}^{(0)}+\mathrm{A}^{(1)} \cos ^{2}\left(\frac{1}{2}\left(\beta^{(0)}+\beta^{(1)}\left(\omega-\omega_{0}\right)+\frac{1}{2} \beta^{(2)}\left(\omega-\omega_{0}\right)^{2}\right) \Delta L\right) .
$$


In this relation, $\beta^{(0)}, \beta^{(1)}$ and $\beta^{(2)}$ denote the propagation constant, its first and its second-order derivative at the center frequency $\omega_{0}$. The parameters $\mathrm{A}^{(0)}, \mathrm{A}^{(1)}, \mathrm{B}^{(0)}=\beta^{(0)} \Delta L, \mathrm{~B}^{(1)}=\beta^{(1)} \Delta L$, and $\mathrm{B}^{(2)}=1 / 2 \beta^{(2)} \Delta L$ are determined by a least-square fit of the model function to the measured transmission spectrum, see Fig. S1. Based on these results, the phase shift $\Delta \phi_{\mathrm{c}}=\left(\frac{1}{2}\left(\beta^{(0)}+\beta^{(1)}\left(\omega_{c}-\omega_{0}\right)+\frac{1}{2} \beta^{(2)}\left(\omega_{c}-\omega_{0}\right)^{2}\right) \Delta L\right)$ is determined for each actual operating point, and used to calculate the associated correction factor $\alpha$. Fig. S1 shows the measured power transmission spectrum of the POH MZM as a function of carrier frequency along with the associated fit according to the Eq. (S3). Note that the electro-optic frequency response shown in Fig. $2 b$ in the main paper is measured in four different segments, covering the frequency band $2 \mathrm{GHz} \leq f_{\mathrm{m}} \leq 64 \mathrm{GHz}$, $0.07 \mathrm{THz} \leq f_{\mathrm{m}} \leq 0.11 \mathrm{THz}, \quad 0.11 \mathrm{THz} \leq f_{\mathrm{m}} \leq 0.17 \mathrm{THz}$ and $0.22 \mathrm{THz} \leq f_{\mathrm{m}} \leq 0.36 \mathrm{THz}$, respectively. Each of these measurements are performed on a slightly modified setup, leading to slightly different operating points that are denoted as $\mathrm{P}_{1}$, $\mathrm{P}_{2}$, $\mathrm{P}_{3}=\mathrm{P}_{2}$, and $\mathrm{P}_{4}$ in Fig. $\mathrm{S} 1$.

To drive the modulator, we use an external RF/THz source that is connected to the un-terminated on-chip coplanar transmission line of the POH MZM using a $50 \Omega$ ground-signal-ground (GSG) probe. In order to estimate the open-circuit peak voltage $U_{\mathrm{e}}$ of the electrical signal that is effective at the POH MZM, we first measure the available electrical power $P_{\mathrm{e}}$ at the input terminals of the GSG probe taking into account losses from various components used in the setup such as cables, connectors, or THz waveguides. For this purpose, we use a calibrated power meter (Anritsu ML2438A) for $2 \mathrm{GHz} \leq f_{\mathrm{m}} \leq 64 \mathrm{GHz}$ and a waveguidecoupled calorimeter (VDi Erickson PM4) for $64 \mathrm{GHz} \leq f_{\mathrm{m}} \leq 0.36 \mathrm{THz}$. We further need to take into account the losses that the THz signal experiences during on-chip propagation along the $150 \mu \mathrm{m}$-long tapered coplanar transmission line from the probe pads to the POH MZM. To this end, we use a commercially available simulation tool (CST Microwave Studio) to calculate the transmission parameter $S_{21, \mathrm{TL}}$ of the tapered transmission line, see green trace in Fig. S2. Moreover, we need to take into account that the line impedance changes along the transmission line taper from an initial value of $Z_{\mathrm{TL} \text {,in }} \approx 50 \Omega$ at the input to a frequency-dependent impedance $Z_{\mathrm{TL} \text {,out }}$ at the output, which is also extracted from the numerical simulation, see blue trace in

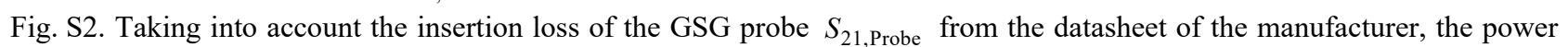
available at the terminals of the POH modulator can be calculated as $P_{\mathrm{POH}}=P_{\mathrm{e}}\left|S_{21, \mathrm{Probe}}\right|^{2}\left|S_{21, \mathrm{TL}}\right|^{2}$. The open-circuit peak drive voltage of the modulating signal at the terminal of the POH MZM is then given by $U_{\mathrm{e}}=2 \sqrt{2 \times Z_{\mathrm{TL}, \mathrm{out}} \times P_{\mathrm{POH}}}$.

To cover the various frequency bands, we use four different GSG probes, which are specified for operation in designated frequency bands: (i) Cascade Microtech Inc. I67-GSG-100 (S/N: HF298) for $2 \mathrm{GHz} \leq f_{\mathrm{m}} \leq 64 \mathrm{GHz}$, (ii) Cascade Microtech Inc. I110-A-GSG-100 (S/N: DV27T) for $0.07 \mathrm{THz} \leq f_{\mathrm{m}} \leq 0.11 \mathrm{THz}$, (iii) Cascade Microtech Inc. I170-T-GSG-100-BT (S/N: $\mathrm{HK} 254$ ) for $0.11 \mathrm{THz} \leq f_{\mathrm{m}} \leq 0.17 \mathrm{THz}$ and (iv) Model 325B from GGB Industries Inc. for $0.22 \mathrm{THz} \leq f_{\mathrm{m}} \leq 0.36 \mathrm{THz}$. The insertion losses $\left(S_{21 \text {,Probe }}\right)$ of the GSG probes are obtained from the respective datasheets. In the frequency range $0.325 \mathrm{THz} \leq f_{\mathrm{m}} \leq 0.36 \mathrm{THz}$, the insertion loss of the THz probe (GGB, Model 325B) is estimated by extrapolating the values from the datasheet, see Fig. S3. Moreover, we need to account for the fact that the drive power $P_{\mathrm{e}}$ from our signal sources shows a strong dependency with respect to the modulation frequency $f_{\mathrm{m}}$ and hence results in a strongly frequency-dependent 


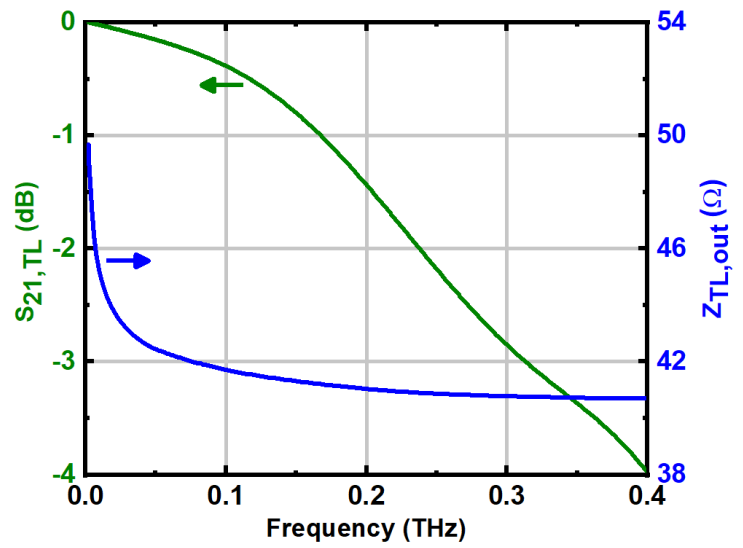

Fig. S2: Simulated RF/THz characteristics of the on-chip tapered transmission line The frequency-dependent insertion loss $S_{21, \mathrm{TL}}$ and line impedance $Z_{\mathrm{TL} \text {,out }}$ of the tapered coplanar transmission line are obtained using CST Microwave Studio

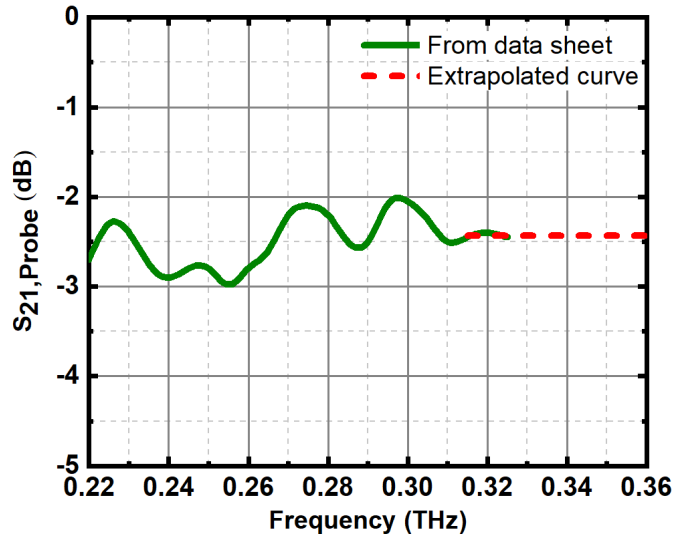

Fig. S3: Insertion loss of the THz GSG probe. $\mathrm{S}_{21}$ parameter of the $\mathrm{THz}$ probe (GGB, Model 325B) as used for calculating the peak electrical drive voltage. The green curve shows the values from the datasheet of the GSG probe and the dashed red curve is an extrapolation in the frequency range between $0.325 \mathrm{THz}$ and $0.36 \mathrm{THz}$. Note that extrapolation by an essentially flat frequency response is a conservative estimation: assuming a frequency-dependent decay of the $S_{21}$ parameter would lead to a decrease of the drive voltage that is assumed to be effective at the modulator. This would result in an increase of the normalized MZM frequency response in Fig. $2 b$ (in the main paper), that is obtained from dividing the measured phase modulation index by the estimated drive voltage amplitude.

modulation index $\eta\left(f_{\mathrm{m}}\right)$. The measured phase modulation index $\eta\left(f_{\mathrm{m}}\right)$ and the calculated electrical drive voltage $U_{\mathrm{e}}\left(f_{\mathrm{m}}\right)$ for each frequency band are shown in Fig. S4(a) - (d). Since the phase modulation index $\eta$ is proportional to the electric drive voltage $U_{\mathrm{e}}$, we can eliminate the impact of the frequency-dependent drive power $P_{\mathrm{e}}\left(f_{\mathrm{m}}\right)$ by considering the ratio $\eta\left(f_{\mathrm{m}}\right) / U_{\mathrm{e}}\left(f_{\mathrm{m}}\right)$ . Normalizing the frequency characteristic of this ratio to its value at a reference modulation frequency of $f_{\mathrm{m}, \mathrm{ref}}=2 \mathrm{GHz}$, we obtain the dimensionless normalized phase modulation index according to the Eq. (1) of the main paper,

$$
\eta_{U}\left(f_{\mathrm{m}}\right)=20 \log _{10}\left(\frac{\eta\left(f_{\mathrm{m}}\right) / U_{\mathrm{e}}\left(f_{\mathrm{m}}\right)}{\eta\left(f_{\mathrm{m}, \mathrm{ref}}\right) / U_{\mathrm{e}}\left(f_{\mathrm{m}, \mathrm{ref}}\right)}\right)
$$

The normalization according to Eq. (S4) allows to infer the global electro-optic (EO) response of the POH MZM measured in various frequency bands, leading to the results shown in Fig. $2 \mathrm{~b}$ in the main paper. From these results it can be observed that the plasmonic MZM has a flat frequency response exceeding $0.36 \mathrm{THz}$ with no sign of bandwidth limitation. Our findings emphasize the fact that the POH modulators are capable of modulating THz frequencies, as predicted in the literature [2], [3], and are in line with previous experiments [4] that demonstrated operation of POH modulators at frequencies up to 0.17 THz. Note, however, that in the work presented in [4], the frequency response was measured in different frequency bands and the results for each band were then independently normalized to the mean of all data points of the specific band. This technique does not relate the measured modulation index to the actual electric drive amplitude in an extended frequency range and hence makes it difficult to infer the overall frequency roll-off of the modulator transfer function. We overcome this problem by using the global normalization technique presented in the preceding paragraphs. 
(a)

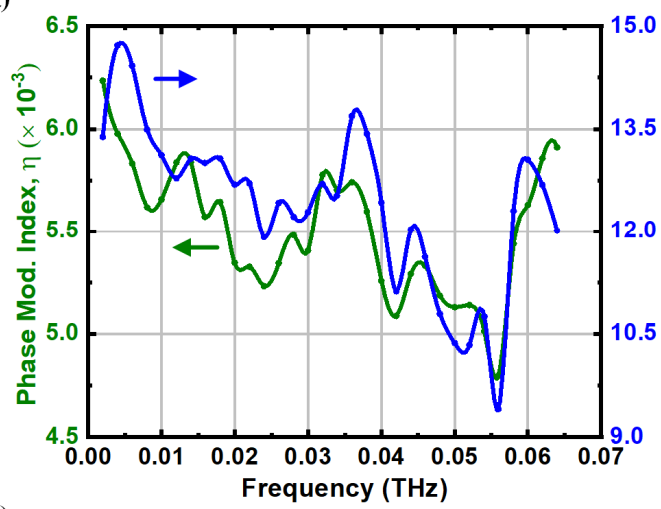

(c)

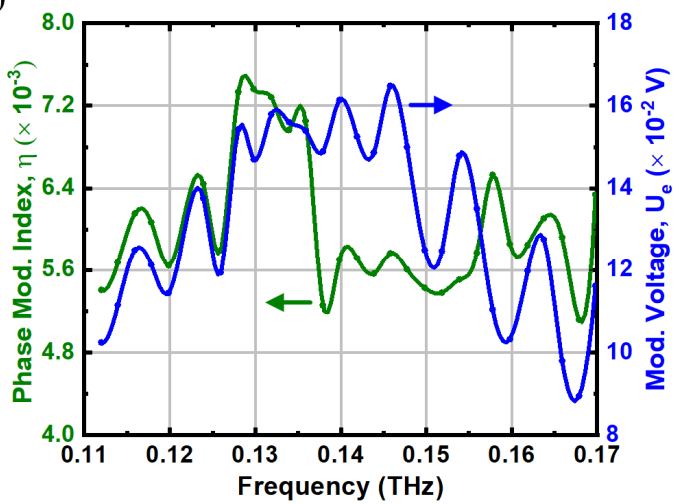

(b)

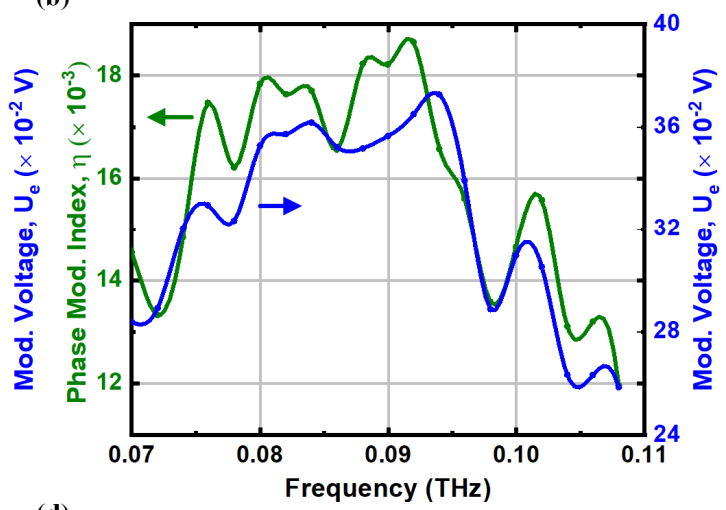

(d)

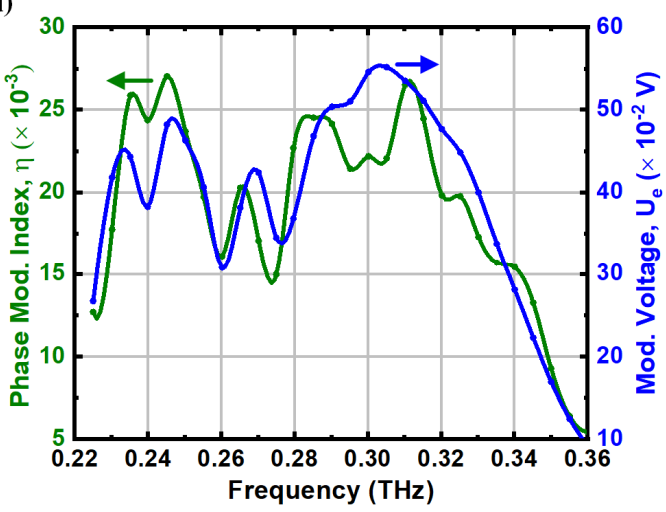

Fig. S4: Frequency-dependent phase modulation indices $\eta$ (green traces) of the MZM and associated voltage amplitudes $\boldsymbol{U}_{\mathrm{e}}$ (blue traces) of the electric drive signals for various frequency bands. (a) $0.002 \mathrm{THz}$ to $0.07 \mathrm{THz}$ ( (b) $0.07 \mathrm{THz}$ to $0.11 \mathrm{THz}$; (c) $0.11 \mathrm{THz}$ to $0.17 \mathrm{THz}$; (d) $0.22 \mathrm{THz}$ to $0.36 \mathrm{THz}$. It can be observed that $\eta$ is essentially proportional to $U_{\mathrm{e}}$, as expected from theory.

The normalized transfer function shown in Fig. $2 b$ of the main paper exhibits noticeable variations of approximately $\pm 2.5 \mathrm{~dB}$ . Such variations can be observed in all our transfer-function measurements, but they vary from measurement to measurement and hence cannot be attributed to an intrinsic behavior of the POH MZM. As an example, Fig. S5 shows the results of an independent measurement of a second-generation POH MZM, which is nominally identical to the first one that is used for the bandwidth measurements shown in Fig. $2 \mathrm{~b}$ of the main paper, except that it relies on YLD124 as an organic EO material [5] rather than on SEO100. The measurement of the second-generation device also shows an EO bandwidth of more than $0.35 \mathrm{THz}$, but also exhibits variations that have approximately the same magnitude but are otherwise not correlated with the ones seen in Fig. $2 b$ of the main paper. We hence attribute these ripples to measurement uncertainties, and not to an intrinsic behavior of the POH MZM.

As a main source of uncertainty, we identify both the measurement of the frequency-dependent $\mathrm{THz}$ drive power and the power measurement of the intensity-modulated optical carrier

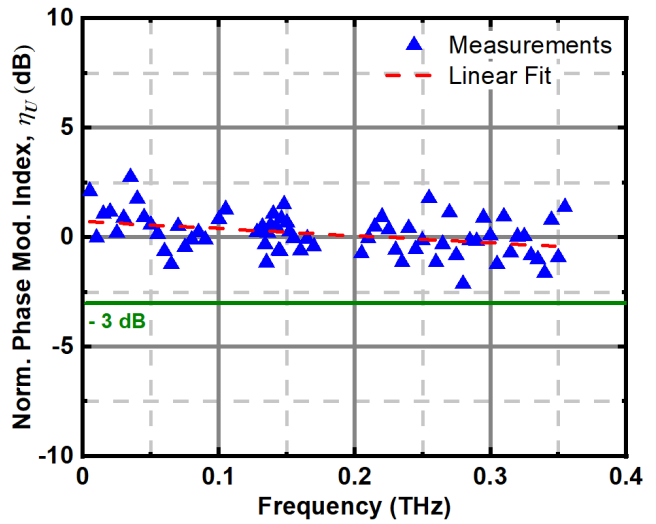

Fig. S5: Normalized phase modulation index $\eta_{U}$ of a secondgeneration POH MZM. The device is based on the nominally same design as in Fig. $2 \mathrm{~b}$ of the main paper, but uses the organic material YLD124 as an EO cladding rather than SEO100. While measurements confirm the ultra-broadband characteristics of a POH MZM, featuring a flat frequency response until at least $0.355 \mathrm{THz}$, it also shows variations that have approximately the same magnitude but are otherwise not correlated with the ones seen in Fig. $2 b$ of the main paper. 
and its sidebands. The THz power was measured with a calorimeter (VDi Erickson PM4, for $f_{\mathrm{m}}>70 \mathrm{GHz}$ ) and is subject to uncertainties due to reflections and limited repeatability of the waveguide flange connections. In addition, we observe a drift of the calorimeter of the order of $10 \ldots 20 \mu \mathrm{W}$ over a few minutes [6], leading to noticeable errors especially for THz power levels below $100 \mu \mathrm{W}$, e.g., in the frequency band from $0.11 \mathrm{THz}$ to $0.17 \mathrm{THz}$. The measurement of the phase modulation index $\eta$, obtained from the sideband-to-carrier power ratio, is also subject to uncertainties because the sideband power levels are small, typically of the order of $-40 \ldots-50 \mathrm{dBm}$, due to the small $\mathrm{THz}$ power of the driving source. The sideband powers are measured with an optical spectrum analyzer (APEX AP2050) having a resolution bandwidth of $180 \mathrm{MHz}$, which was necessary to suppress amplified spontaneous emission (ASE) noise originating from the erbium-doped fiber amplifier (EDFA) used in the experiment. When repeatedly scanning the same optical sideband, we observe power variations of up to $\pm 1 \mathrm{~dB}$, which translate directly into similar variations of the estimated modulation index $\eta_{U}$, see Eq. (S4). Moreover, small ripples in the optical transfer function due to reflections between the on-chip optical grating couplers may also influence the measured optical sideband power and therefore the extracted $\eta_{U}$. All these effects contribute to the measured ripples in the frequency response of the POH MZM. Additional uncertainties may arise from the THz transmission lines used to feed the modulator. These transmission lines comprise several connectors and the hollow waveguide sections with tapers and flanges, where small reflections and hence ripples in the transfer function could occur.

Note that the POH MZM used for the bandwidth measurement shown in Fig. 2 of the main paper is a first-generation device with non-optimized on-chip passive components. The overall fiber-to-fiber insertion loss of the device amounts to about $38 \mathrm{~dB}$, measured at the highest transmission of the grating couplers. This comprises a $12 \mathrm{~dB}$ intrinsic insertion loss of the POH phase shifters, a $24 \mathrm{~dB}$ of loss that originates from non-optimum grating couplers ( $12 \mathrm{~dB}$ per coupler), and an additional loss of $2 \mathrm{~dB}$ due to on-chip waveguides and multi-mode interference (MMI) couplers. Moreover, a non-optimum poling of the EO polymer (SEO100) resulted in a rather high $\pi$-voltage of this device (about $30 \mathrm{~V}$ ), while the available wideband-tuneable electric sources used for driving the devices typically deliver rather small on-chip powers $P_{\mathrm{POH}}$ down to $25 \mu \mathrm{W}$. This corresponds to on-chip voltage swings with amplitudes $U_{\mathrm{e}}$ of $0.1 \mathrm{~V}$, leading to sideband-to-carrier power ratios of $-52 \mathrm{~dB}$ and phase modulation indices as small as $5 \times 10^{-3} \mathrm{rad}$. In combination with the $38 \mathrm{~dB}$ optical insertion loss, this leads to weak sidebands that are $90 \mathrm{~dB}$ below the optical input power of around $18 \mathrm{dBm}$, as measured in the fiber just before the modulator. For detection of these small sidebands powers in our bandwidth measurement experiment, we use an EDFA with a gain of $30 \mathrm{~dB}$ in combination with a highresolution $(180 \mathrm{MHz})$ optical spectrum analyzer to effectively suppress the ASE noise of the EDFA. Note that there is a vast potential to considerably reduce the $\pi$-voltage of the POH MZM, see Section $\mathrm{V}$, and also the overall insertion loss of the device, see Section VI. As an example, we rely on grating couplers with reduced etch depth for the second generation of our devices, leading to an insertion loss of $6.5 \mathrm{~dB}$ per fiber-to-chip interface - much lower than the $12 \mathrm{~dB}$ measured for our first-generation devices. This was not only an important step towards the data transmission experiments shown in Fig. 3 of our main paper, but also allowed us to omit the EDFA for subsequent bandwidth measurements that are shown in Fig. S5.

\section{Characterization of the uni-travelling-carrier photodiode and the THz amplifiers}

The frequency response of the uni-travelling-carrier photodiode (UTC-PD) employed in the data transmission experiments is characterized using the setup depicted in Fig. S6(a). Light from two detuned external-cavity lasers (ECL, Agilent N7714A) emitting identical polarizations with similar optical powers at frequencies $f_{\mathrm{a}}=193.0 \mathrm{THz}$ and $f_{\mathrm{b}}=193.3 \mathrm{THz}$ is combined in a polarization maintaining $3 \mathrm{~dB}$ coupler. The superimposed signal is amplified to an optical power $P_{\text {opt }}$ and then fed to the UTC photodiode for photomixing to generate a continuous-wave $(\mathrm{CW}) \mathrm{THz}$ signal at a frequency $f_{\mathrm{THz}}=\left|f_{\mathrm{a}}-f_{\mathrm{b}}\right|=0.3 \mathrm{THz}$. A polarization controller (PC) is used to adjust the polarization of the superimposed beat signal for maximizing THz output signal of the polarization-dependent UTC-PD. The frequency of the CW THz signal is tuned from $0.25 \mathrm{THz}$ to $0.33 \mathrm{THz}$ by varying the optical frequency $f_{\mathrm{a}}$, and the resulting THz output power $P_{\mathrm{THz}}$ is measured with a waveguide-coupled calorimeter (VDi, Erickson PM4). Fig. S6(b) shows the measured THz output power as a function of the generated THz frequency $f_{\mathrm{THz}}$ for an input optical power of $P_{\mathrm{opt}}=16 \mathrm{~mW}$, which generates a direct photocurrent of $I_{\mathrm{ph}}=5.6 \mathrm{~mA}$ in the reverse-biased UTC -PD. 
(a)

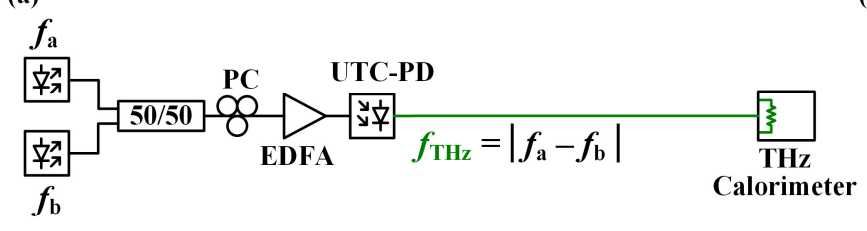

(c)

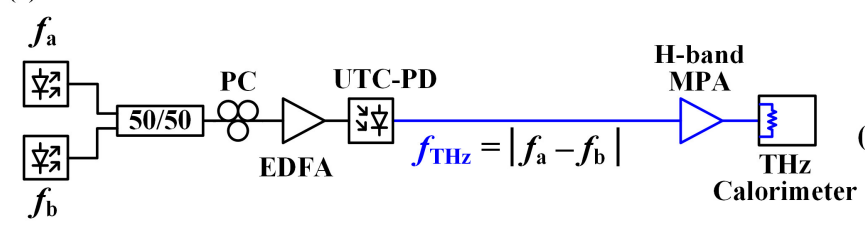

(b)

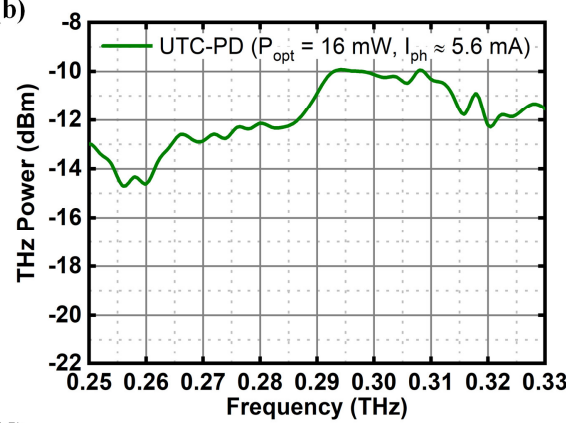

(d)

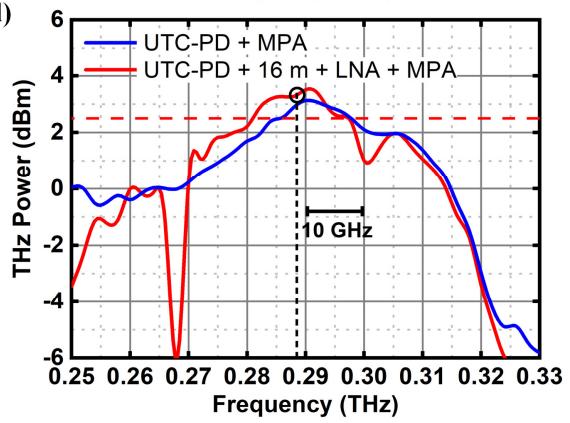

Fig. S6: Setup for characterizing various THz components used in the data transmission experiment. For all the measurements, the optical input power to the uni-travelling-carrier photodiode (UTC-PD) is set to $P_{\mathrm{opt}}=16 \mathrm{~mW}$ which corresponds to a direct photocurrent of $I_{\mathrm{ph}}=5.6 \mathrm{~mA}$. (a) Setup for measuring the THz output power of the UTC-PD (b) Frequency-dependent THz power of UTC-PD as measured by calorimeter (c) Setup for measuring the available THz power after amplification by H-band medium-power amplifier (MPA). (d) Frequency-dependent THz power for the combination of UTC-PD and MPA (blue curve). (e) Setup for measuring the THz power available after a free-space transmission distance of $16 \mathrm{~m}$ and a cascaded amplification stage, comprising a low-noise amplifier (LNA) and the MPA. The available THz power as a function of frequency is shown as the red curve in (d). The kinks in the red curve are attributed to imperfect waveguide connector flanges. A horizontal red dashed line indicates a minimum $\mathrm{THz}$ power of $2.5 \mathrm{dBm}$ that was needed at $20 \mathrm{GBd}$ to achieve a BER of $4.5 \times 10^{-3}$, corresponding to the threshold for forward-error correction (FEC) with $7 \%$ coding overhead, see Fig. $3 \mathrm{~d}$ in the main paper. The operating frequency of the THz communication link for the data transmission experiments is marked with a circle and a vertical line.

Next, we add an H-band medium-power amplifier (MPA, M145AMPH) with a $15 \mathrm{~dB}$ gain at $0.3 \mathrm{THz}$ and a saturation output power $+4 \mathrm{dBm}$ to the setup to measure the total available THz power after amplification, see Fig. S6(c). The THz amplifier is a millimeter-wave monolithic integrated circuit (MMIC) based on metamorphic high-electron-mobility transistor (mHEMT) technology [7]. Fig. S6(d) shows the measured THz power as a function of frequency for the combination of UTC-PD and MPA (blue curve). Finally, we use the setup depicted in Fig. S6(e) to measure the available THz power after a free-space transmission of $16 \mathrm{~m}$. The UTC-PD transmitter is connected to a horn antenna ( $26 \mathrm{dBi}$ gain), which radiates the THz signal into free-space. A polytetrafluoroethylene (PTFE) lens is used to collimate the beam that is emitted by the horn antenna. At the receiver, a similar PTFE lens focuses the THz signal to another horn antenna (26 dBi gain). A low-noise H-band amplifier (LNA, M145ALNH) with a gain of $26 \mathrm{~dB}$ at $0.3 \mathrm{THz}(9 \mathrm{~dB}$ noise figure, $-30 \mathrm{dBm}$ maximum input power) cascaded with the previously mentioned H-band MPA is used to compensate the free-space loss of the THz signal. The red curve in the Fig. S6(d) shows the THz available power at the receiver after a transmission distance of $d=16 \mathrm{~m}$ and subsequent amplification by the combination of LNA and MPA. The dips in the red curve are attributed to reflections of the THz carrier due to imperfect waveguide connector flanges between the amplifiers. The operating frequency $f_{\mathrm{Tx}, \mathrm{THz}}=0.2885 \mathrm{THz}$ of the $\mathrm{THz}$ data transmission experiments is marked with a circle and a dashed vertical line. In our experiments, the free-space path loss (FSPL) of the wireless link would amount to approximately $106 \mathrm{~dB}$ according to the relation $\mathrm{FSPL}=10 \log _{10}\left(c / 4 \pi f_{\mathrm{Tx}, \mathrm{THz}} d\right)^{2}$ where $c$ is the vacuum speed of light. From the measurements of the received power, we estimate that the combination of transmitter-receiver lens-and-horn antenna feature a gain of $\sim 79 \mathrm{dBi}$ compared to an isotopic antenna, see Section III. 
In the transmission experiment, Fig. 3 of the main paper, a minimum THz power of approximately $2.5 \mathrm{dBm}$ was needed at $20 \mathrm{GBd}$ to achieve a BER of $4.5 \times 10^{-3}$, corresponding to the threshold for forward-error correction (FEC) with $7 \%$ coding overhead. This power level refers to the waveguide input of the THz GSG probe and was estimated by taking the minimum THz power within the spectral range occupied by the $20 \mathrm{GBd}$ signal, which is indicated a dashed horizontal line in Fig. S6(d). Taking into account the GSG probe losses depicted in Fig. S3, this corresponds to an on-chip THz drive power of approximately $0 \mathrm{dBm}$. Note that the THz power level required to reach the FEC threshold and the associated signal-to-noise power ratio (SNR) increases in proportion to the symbol rate.

\section{Setup for data transmission experiments}

A detailed sketch of the THz transmitter (Tx) for optical-to- $\mathrm{THz}(\mathrm{O} / \mathrm{T})$ conversion is depicted in Fig. S7(a). A software-driven digital-to-analog converter (DAC, Keysight M9505A, sampling frequency $90 \mathrm{GSa} \mathrm{s}^{-1}$ ) is used to generate drive signals for QPSK modulation using a pseudo-random binary sequence (length $2^{11}-1$ ). The signals are shaped to Nyquist pulses featuring a raisedcosine spectrum and a roll-off factor of $\beta=0.1$. The resulting analog signal is fed to an optical IQ-modulator that is fed by a $\mathrm{CW}$ carrier generated by an external-cavity laser (ECL) at an optical frequency $f_{0}=193.3 \mathrm{THz}$. The IQ-modulator is biased at its zero-transmission point in order to suppress the optical carrier at frequency $f_{0}$. The intensity-modulated optical signal is amplified using an erbium-doped fiber amplifier (EDFA). Out-of-band amplified spontaneous emission noise from the EDFA is blocked by an optical band-pass filter ( $0.6 \mathrm{~nm}$ passband width) before superimposing the modulated optical carrier with a $\mathrm{CW}$ local-oscillator (LO) tone at a frequency $f_{\mathrm{Tx}, \mathrm{LO}}$ derived from another ECL. The power of the CW tone at $f_{\mathrm{Tx}, \mathrm{LO}}$ is adjusted to the same level as the average power of the modulated signal. The combined signal is further amplified by a second EDFA, polarization-adjusted, and then fed into a variable optical attenuator (VOA). The CW tone and the optical data signal are mixed

in a UTC-PD, resulting in a THz QPSK data stream at a carrier frequency $f_{\mathrm{Tx}, \mathrm{THz}}=\left|f_{0}-f_{\mathrm{Tx}, \mathrm{LO}}\right|=0.2885 \mathrm{THz}$. The polarization of the superimposed signal entering the polarization-sensitive UTC-PD is adjusted to maximize the THz output power. This THz transmitter implementation provides the flexibility of tuning the THz carrier frequency $f_{\mathrm{Tx}, \mathrm{THz}}$ over a broad frequency range, only limited by the bandwidth of the UTC-PD, by adjusting the frequency $f_{\mathrm{Tx}, \mathrm{LO}}$ of the unmodulated LO tone. The THz output power provided by the UTC-PD typically amounts to $-11 \mathrm{dBm}$. A conical horn antenna with a gain of $26 \mathrm{dBi}$ and an aperture diameter of $5.6 \mathrm{~mm}$ is attached directly to the UTC-PD to radiate the THz signal into free-space. The radiated signal is collimated by a plano-convex PTFE lens (diameter - $101.6 \mathrm{~mm}$ and focal length - $200 \mathrm{~mm}$ ) and is sent over a wireless transmission distance of $16 \mathrm{~m}$.

Fig. S7(b) shows the THz receiver (Rx) setup used for THz-to-optical (T/O) conversion. Light emitted by an ECL at a frequency $f_{\mathrm{Rx}, \mathrm{LO}}$ is amplified to a power of $18 \mathrm{dBm}$ by an EDFA and then launched into the POH MZM using an on-chip grating coupler on the silicon ( $\mathrm{Si}$ ) photonic chip. The on-chip insertion loss of the MZM with $20 \mu \mathrm{m}$-long POH phase shifter amounts to $16 \mathrm{~dB}$. The grating couplers and the on-chip waveguides contribute to an additional loss of $14 \mathrm{~dB}$, with vast potential for further reduction by using optimized passive silicon photonic devices. The passive silicon photonic waveguides feeding the POH MZM have a length difference of $80 \mu \mathrm{m}$ and hence enable the adjustment of the quadrature $(3 \mathrm{~dB})$ operating point of the $\mathrm{POH} \mathrm{MZM}$ by tuning the wavelength. Alternatively, the operating point of the POH MZM could be set by applying a DC bias or by additional phase shifters in the silicon photonic waveguides of the MZM arms. For wireless reception, the THz QPSK data stream is focused to a horn antenna (26 dBi gain) by a PTFE plano-convex lens similar to the one used in the THz transmitter setup. A cascaded combination of H-band LNA and MPA boosts the power of the THz signal and drives the POH MZM through a THz GSG probe (GGB, Model 325B). The intensity-modulated signal out of the plasmonic modulator is coupled out of the chip using another grating coupler and then amplified by an EDFA. One of the modulated sidebands of the amplified signal at a frequency $f_{\mathrm{Rx}, \mathrm{LO}} \pm f_{\mathrm{Tx}, \mathrm{THz}}$ is filtered with a $0.6 \mathrm{~nm}$ band-pass filter and sent through a second amplification stage, where the optical signal is further amplified to a power of $1 \mathrm{dBm}$. The resulting signal is again filtered with a $1 \mathrm{~nm}$ band-pass filter to additionally suppress the optical carrier and one of the modulation sidebands before being detected by a coherent optical receiver (Agilent optical modulation analyzer N4391A, OMA). The received electrical signal is recorded by a real-time oscilloscope (32 GHz 


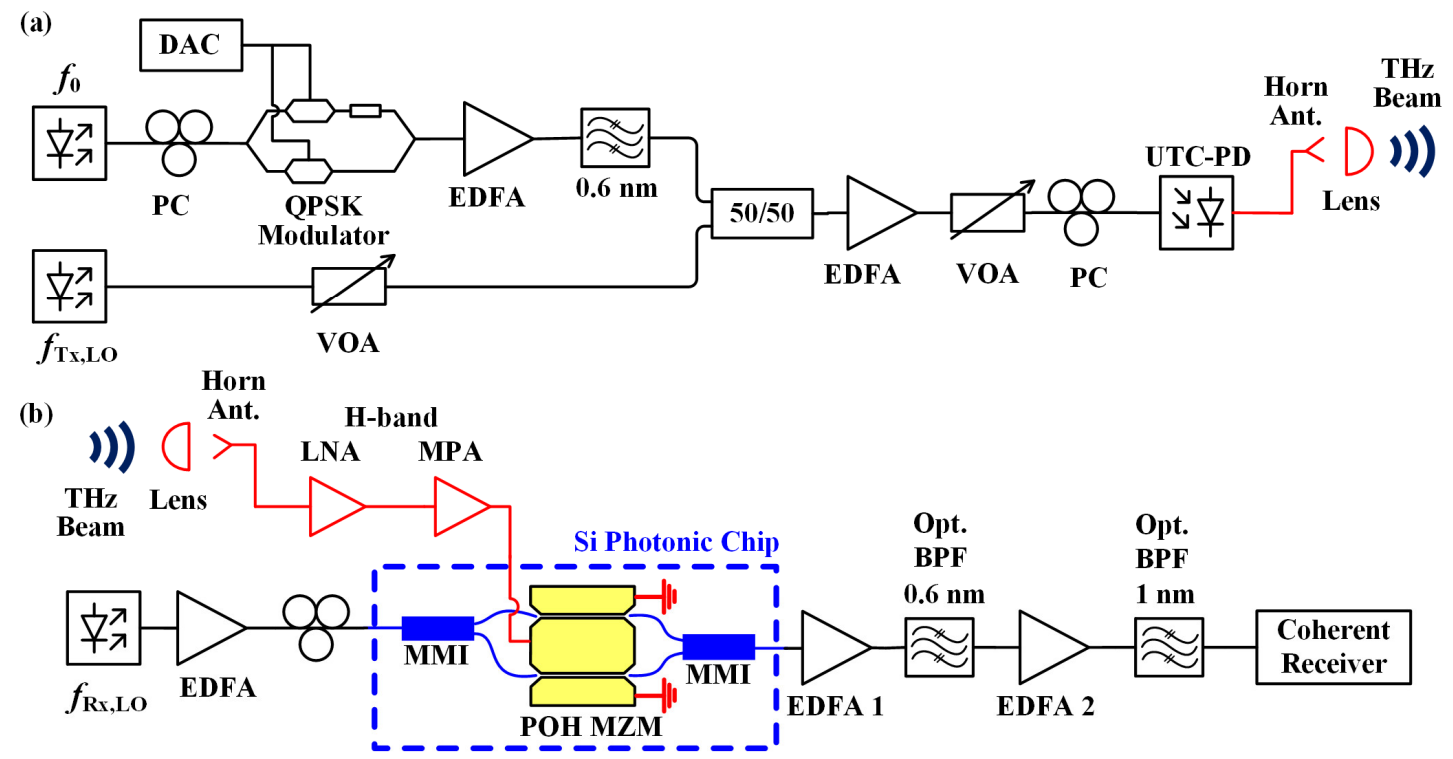

(c)

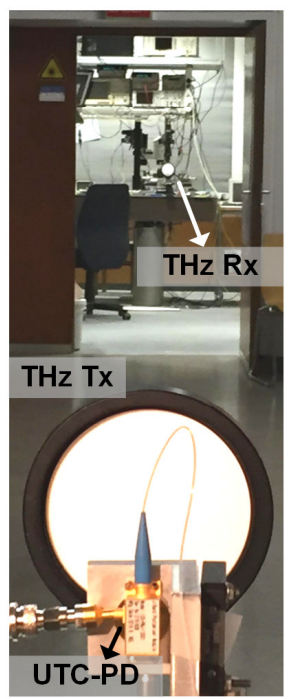

(d)

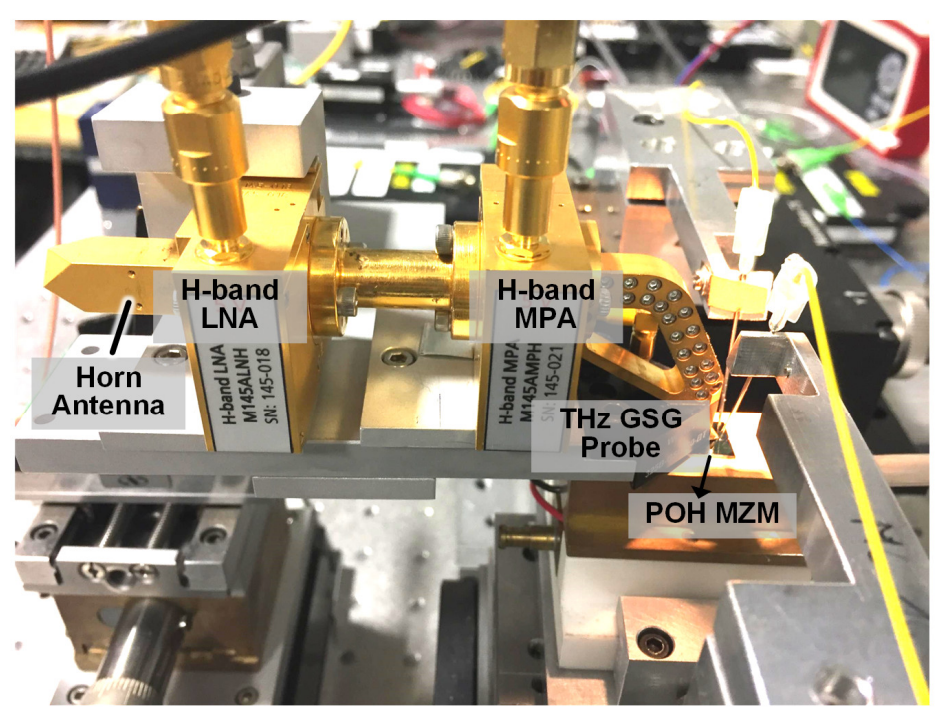

Fig. S7: Detailed setup of the THz transmitter and receiver used for data transmission experiments. (a) Setup of the wireless terahertz transmitter that generates a QPSK data signal on a carrier frequency of $f_{\mathrm{Tx}, \mathrm{THz}}=0.2885 \mathrm{THz}$ by exploiting photomixing in a uni-travelling-carrier photodiode (UTC-PD) for optical-to- $\mathrm{THz}(\mathrm{O} / \mathrm{T})$ conversion. The THz signal is radiated into free-space by a horn antenna. A subsequent polytetrafluoroethylene (PTFE) lens is used to collimate the THz beam. (b) Setup for terahertz-tooptical (T/O) conversion at the THz receiver using a plasmonic Mach-Zehnder modulator (MZM). The incoming signal is focused into a horn antenna by a second PTFE lens. It is then fed to a cascade of $\mathrm{THz}$ (H-band) amplifiers, which drive the POH MZM, thereby modulating the signal onto an optical carrier. A cascade of EDFA and optical band-pass filters (BPF) is used to amplify the intensity-modulated optical signal and to isolate one of the sidebands. Note that, due to the limited tuning range of the optical filter used in this experiment, we chose to isolate the lower sideband of our optical signal rather than the upper one, as sketched in Fig.1c of the main paper. (c) Photograph of the data transmission setup showing the THz Tx and the Rx. Tx and Rx are built up in different laboratories, separated by $16 \mathrm{~m}$ of free-space transmission distance across the hallway. (d) Photograph of the THz photonic Rx. The wireless signal is focused by a PTFE lens (not shown), then coupled into the horn antenna and amplified by the dual-stage $\mathrm{H}$-band amplifier, which drive the POH MZM through the ground-signal-ground (GSG) probe. DAC - digital-to-analog converter, PC - polarization controller, MMI - multimode interference coupler, EDFA - erbium-doped fiber amplifier, VOA - variable optical attenuator, LNA - low-noise amplifier, MPA - medium-power amplifier.

analog bandwidth) and evaluated off-line using digital signal processing (DSP). After resampling and clock-recovery, the recorded data is equalized using a constant-modulus algorithm. The phase of the carrier is estimated based on a Viterbi-Viterbi algorithm, and the bit error ratio (BER) is computed as a final step. 
Fig. S7(c) shows a photograph of the THz communication link with the transmitter and receiver separated by $16 \mathrm{~m}$. The THz beam path contains a horn antenna and a plano-convex PTFE lens on each side. Fig. S7(d) shows a photograph of the THz receiver, comprising a horn antenna and a combination of H-band LNA and MPA which drive the POH MZM through a THz ground-signal-ground (GSG) probe. From the free-space path loss (FSPL) of approximately $106 \mathrm{~dB}$, see Section II, we estimate that the directivity gain of each antenna-lens combination is approximately $40 \mathrm{dBi}$. We later realized that the insertion loss of the $\mathrm{THz}$ beam path could be further reduced by more careful alignment of the components, leaving room for further increasing the performance of the transmission link.

\section{Signal impairment analysis}

In contrast to conventional optical transmission links, the quality of the $\mathrm{T} / \mathrm{O}$-converted signal in our demonstration is dictated by the quality of the modulating THz signal and not so much by the optical transmission link. Specifically, the THz signal might be subject to impairments like frequency-dependent losses of the free-space path, electrical noise of the THz amplifiers, or bandwidth limitations of the $\mathrm{O} / \mathrm{T}$ and $\mathrm{T} / \mathrm{O}$ converters. Due to these fundamental differences to classical fiber-optic transmission schemes, it is difficult to compare the constellation diagrams shown in Fig. $3 \mathrm{e}$ of our main paper with those of conventional optical transmission links. In the following, we present a detailed analysis of our data transmission demonstration and the corresponding results are shown in Fig. S8.

For high symbol rates, we find that the dominant signal impairments are introduced when amplifying the weak optical output signal of the POH MZM by an erbium-doped fiber amplifier, which is labelled as EDFA 1 in Fig. S7(b). The signal-to-noise power ratio (SNR) at the optical receiver can be calculated from the ratio of the optical signal power $P_{\mathrm{s}}$ and the noise power density $N_{0}$ within the signal bandwidth $B$, and the error vector magnitude (EVM) can be estimated assuming data-aided reception according to the relation [8]-[10]

$$
\mathrm{SNR}=\frac{P_{\mathrm{S}}}{N_{0} B}, \quad \mathrm{EVM} \approx(\mathrm{SNR})^{-1 / 2}
$$

In this relation, $N_{0}$ denotes the power spectral density of the amplified spontaneous emission (ASE) background that is copolarized with the signal. We estimated the BER of the QPSK signal from the estimated data-aided EVM using the (corrected [9]) Eq. (4) in [8],

$$
\mathrm{BER}=\frac{1}{2} \operatorname{erfc}\left(\sqrt{\frac{1}{2 \mathrm{EVM}^{2}}}\right) \approx \frac{1}{2} \operatorname{erfc}\left(\sqrt{\frac{\mathrm{SNR}}{2}}\right) .
$$

Note that the BER estimation according to Eq. (S6) relies on the assumption that the signal is dominantly impaired by additive white Gaussian noise (AWGN). It can be deduced from Fig. 3b in Ref. [8] that the BER estimate according to Eq. (S6) is reliable for $10^{-5} \leq \mathrm{BER} \leq 10^{-2}$ but the BER is increasingly under-estimated and hence unreliable for BER $\leq 10^{-5}$ as the difference in the EVM between data-aided and non-data-aided reception becomes significant [8]. The results of this reliable estimation of BER are depicted as filled blue circles in Fig. S8(a) while the under-estimated and unreliable BER is represented by open blue circles. For high symbol rates, the BER estimate is consistent with the BER that we actually measure in our experiments, indicated as red triangles in Fig. S8(a). This shows that the ASE background introduced by EDFA 1 is the main source of signal impairment. For low symbol rates, however, the directly measured BER is much higher than the (unreliably) estimated ones from the SNR. This is attributed to additional signal impairments that cannot be described only by an AWGN background.

To understand these additional impairments, we investigate the signal spectra as well as the QPSK constellation diagrams for signals with smaller symbol rates. As an example, the signal spectrum and the respective constellation diagram for a 4-GBd QPSK signal after T/O conversion and coherent optical detection are shown in Fig. S8(b) and (c). The spectrum exhibits an additional "pedestal", encircled with broken red lines, Fig. S8(b), which peaks out of the white-noise background. We attribute this pedestal to nonlinear distortions caused by third-order intermodulation products in the THz amplifiers that were operated close to their saturation output power. These impairments are also present at higher symbol rates where the signal spectrum is 

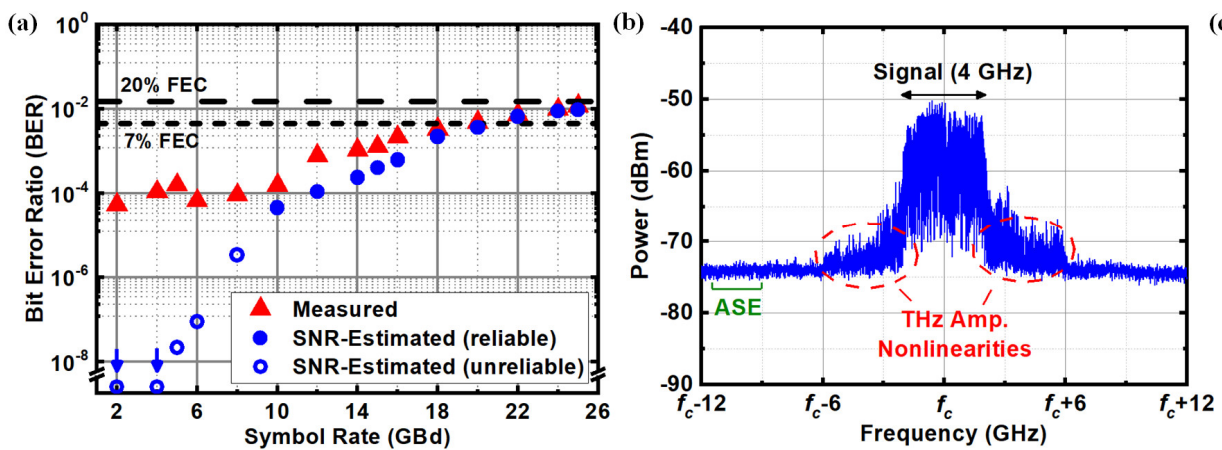

(c)

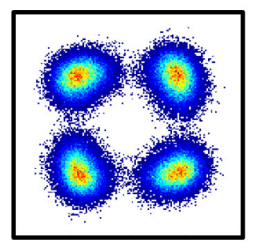

QPSK, $f_{\text {sym }}=4$ GBd

Fig. S8: BER and signal impairment analysis. (a) Bit error ratio (BER) of QPSK signals at different symbol rates upon T/O conversion and coherent optical reception. The red triangles denote the measured BER, while the filled blue circles represent BER values that can be reliably estimated from the SNR of the received signal according to Eq. (S6). The open blue circles correspond to SNR-based estimations that become increasingly unreliable for BER $\leq 10^{-5}$. For high symbol rates, the measured BER fits well with the theoretical estimations, indicating that the SNR is limited by the ASE noise of the EDFA. For smaller symbol rates, however, the measured BER differs strongly from the (unreliably) estimated ones, due to nonlinearities of the THz amplifiers and due to phase-noise of the LO laser used in the coherent Rx. (b) Spectrum showing nonlinear signal impairments of a 4 GBd QPSK signal at the coherent receiver. The spectrum exhibits noticeable 'pedestals' on both sides of the actual data signal, caused by nonlinearities of the THz amplifiers, which are operated close to their saturation output power. (c) Constellation map of the 4 GBd QPSK signal with elongated constellation points indicating phase noise from the free-running laser used for coherent reception.

wider and has a smaller power spectral density. In this case, however, the nonlinearity-generated spectral parts have a correspondingly reduced spectral power density and are therefore masked by the white-noise background. Moreover, despite the use of advanced phase tracking algorithms in our post-processing routines, the signals at low symbol rates are impaired also by phase noise, which can be inferred from the azimuthally elongated constellation points in Fig. S8(c). This phase noise originates from the finite coherence time of the free-running LO laser used for coherent reception of the QPSK signal and its impact increases with decreasing symbol rate [11].

Note that, both for low and high symbol rates, the BER is essentially independent of the length of the optical transmission link in the signal path, since the ASE noise background and the nonlinear distortions at the output EDFA 1 are already very strong. Hence, the signal quality will not significantly degrade by ASE noise introduced by additional EDFA along the optical link. The insertion loss as well as the $\pi$-voltage of the POH MZM can be significantly reduced, see Section V, which directly corresponds to an improved signal quality.

In a back-to-back experiment, we used only the medium-power THz amplifier (MPA), resulting in drive powers that are smaller than those of the transmission experiment, see blue curve in Fig. S6(d), and expected to see a smaller BER owing to the smaller influence of amplifier saturation. However, due to a degradation in the $\pi$-voltage of the unsealed modulator, the measured BER showed much poorer performance than that of the transmission experiment.

\section{POH modulator performance and potential for improvements}

The POH MZM described in the main paper is an experimental device that still leaves significant room for further performance improvements. The following section presents various performance metrics and the underlying theoretical limits, and discusses the path for future improvements.

In general, plasmonic-organic hybrid (POH) modulators stand out due to an ultra-small $\pi$-voltage-length product $U_{\pi} L$. This is a consequence of the strong optical confinement within the nano-scale slot, and of the high EO activity of the organic cladding materials [2], [12]. The $\pi$-voltage-length product of a push-pull MZM is calculated according to Eq. (7) in [2] as 


$$
U_{\pi} L=\frac{w \lambda_{c}}{2 n_{\mathrm{EO}}^{3} r_{33} \Gamma_{\text {slot }}},
$$

where $\lambda_{c}=1.55 \mu \mathrm{m}$ is the operating wavelength, $w$ is the slot-width, $\Gamma_{\text {slot }}$ is the field interaction factor describing the interaction between the modulating RF field and the optical field, and $n_{\mathrm{EO}}^{3} r_{33}$ is the EO figure-of-merit. In the device used in our transmission experiments, the $U_{\pi} L$ product amounts to $240 \mathrm{~V} \mu \mathrm{m}$, which is been achieved with a slot width of $75 \mathrm{~nm}$, a field interaction factor of $\Gamma_{\text {slot }}=0.77$, and a moderate electro-optic coefficient of $r_{33}=64 \mathrm{pm} / \mathrm{V} \quad\left(n_{\mathrm{EO}}^{3} r_{33}=315 \mathrm{pm} / \mathrm{V}\right)$. This leaves vast room for improvement: the field interaction factor can be increased to values [2] of approximately 0.9 by using a metal thickness of $180 \mathrm{~nm}$ along with — technically still feasible — slot widths down to $60 \mathrm{~nm}$. In addition, organic EO materials have recently been demonstrated to exhibit EO coefficients in excess of $r_{33}=390 \mathrm{pm} / \mathrm{V} \quad\left(n_{\mathrm{EO}}^{3} r_{33}=2300 \mathrm{pm} / \mathrm{V}\right)$, enabled by theoryguided molecular optimization of EO chromophores [13]. Together, this would reduce the $\pi$-voltage-length product to values below $35 \mathrm{~V} \mu \mathrm{m}$ at a wavelength of $1.55 \mu \mathrm{m}$, corresponding to a seven-fold improvement compared to our current device. The feasibility of devices with $U_{\pi} L$ products down to an impressive $50 \mathrm{~V} \mu \mathrm{m}$ at $\lambda_{\mathrm{c}}=1.55 \mu \mathrm{m}$ has been previously demonstrated [14], but these devices were not tested with respect to high-frequency modulation.

Ultra-small $U_{\pi} L$ products are also of highest importance when it comes to optical insertion loss - one of the most crucial performance limitations of $\mathrm{POH}$ modulators. Since the plasmonic devices suffer from significant optical propagation losses in the metal slot waveguide, the phase shifter section has to be kept short, typically with lengths of the order of a few tens of micrometers. This leads to a fundamental trade-off between low insertion loss and low $\pi$-voltage $U_{\pi}$, which can be quantified by the product of the propagation loss $a$, expressed in $\mathrm{dB} / \mu \mathrm{m}$, and the $U_{\pi} L$ product in $\mathrm{V} \mu \mathrm{m}$, resulting in a $\pi$-voltage-loss product $a U_{\pi} L$, expressed in $\mathrm{V} \mathrm{dB}$. Our current POH MZM features a $U_{\pi} L$ product of $240 \mathrm{~V} \mu \mathrm{m}$ and a propagation loss of $a=0.8 \mathrm{~dB} / \mu \mathrm{m}$ corresponding to a $a U_{\pi} L$ product of about $190 \mathrm{~V} \mathrm{~dB}$. The propagation loss may be reduced to about $0.2 \mathrm{~dB} / \mu \mathrm{m}$ by using optimized metal deposition techniques [15] and by resorting to alternative materials which feature smaller plasmonic losses, for example silver. Together with the aforementioned possible reduction of the $U_{\pi} L$ product to $35 \mathrm{~V} \mu \mathrm{m}$, this would permit more than a 25 -fold improvement of the $a U_{\pi} L$ product, leading to values of the order of $7 \mathrm{~V} \mathrm{~dB}$. Note that these $a U_{\pi} L$ products are even smaller than those of state-of-the-art silicon depletion-type modulators, which typically [16], [17] exceed $10 \mathrm{~V} \mathrm{~dB}$.

The most attractive feature of POH modulators is the ultra-high modulation bandwidth, which is enabled by the small device footprint and by the ultra-small capacitance, which is of the order of a few fF. Assuming a signal source with a $50 \Omega$ internal impedance and a $3 \mathrm{fF}$ capacitance as estimated for our device, this would lead to RF cutoff frequency [2] in excess of $1 \mathrm{THz}$. This is in good agreement with the bandwidth measurements presented in Fig. $2 b$ of the main paper. These measurements do not show any sign of frequency roll-off, and are also well in line with published [4] and unpublished [18] findings from other research groups. The signal bandwidth demonstrated in our experiment may hence be further increased by at least a factor of two.

\section{General considerations for seamless interfacing of wireless $\mathrm{THz}$ links and fiber-optic net- works}

With respect to the integration of current $\mathrm{POH}$ devices into fiber-optic networks, the optical losses are still too high, even when considering the second device generation which features a fiber-to-fiber insertion loss of about $26 \mathrm{~dB}$ (comprising $6.5 \mathrm{~dB}$ of loss per grating coupler, $12 \mathrm{~dB}$ loss of the plasmonic waveguides, and $1 \mathrm{~dB}$ loss of on-chip waveguides and non-optimum MMI couplers). The loss of the on-chip grating couplers can be reduced to below $2 \mathrm{~dB}$ per coupler [19], and further down to 0.7 dB by incorporating reflecting surfaces underneath the grating couplers [20]. Moreover, the intrinsic loss of the $20 \mu \mathrm{m}-$ long plasmonic phase shifters can be reduced to $4 \mathrm{~dB}$ by using silver as a low-loss plasmonic material, see Section V. Implementing these improvements brings down the total insertion loss to less than $6 \mathrm{~dB}$, comprising $1.4 \mathrm{~dB}$ for the grating couplers and $4 \mathrm{~dB}$ for the $20 \mu \mathrm{m}$-long POH MZM. This compares well with the insertion losses of state-of-the-art silicon depletion-type modulators [16] that are typically used in optical communications, but that are far from providing the required modulation bandwidths in excess of $0.3 \mathrm{THz}$. Note, however, that even the non-optimum POH MZM used in our current THz transmission experiments would 
permit transmission of the optical signal over tens or even hundreds of kilometers of optical fiber. This is due to the fact that the optical signal-to-noise ratio (OSNR) is dominated by the ASE noise of the first EDFA after the POH MZM and hence any reamplification along the fiber link would not deteriorate the OSNR significantly.

In the current proof-of-concept demonstrations, the POH MZM is driven with a THz power of $0 \mathrm{dBm}$, see Section II, and the transmission distance of the THz wireless link is $16 \mathrm{~m}$, limited by the width of the building in which the experiment was performed. This span is still smaller than those envisaged for medium-distance links, in which wireless signal should be transmitted over of $100 \mathrm{~m} \ldots 1 \mathrm{~km}$ [21]. For a distance $d=16 \mathrm{~m}$ bridged in our current transmission experiment, the free-space path loss

(FSPL) at a carrier frequency of $f_{\mathrm{Tx}, \mathrm{THz}}=0.2885 \mathrm{THz}$ is $10 \log _{10}\left(c / 4 \pi f_{\mathrm{Tx}, \mathrm{THz}} d\right)^{2}=106 \mathrm{~dB}$, and the THz transmitter power amounts to $-11 \mathrm{dBm}$, measured at the output of the UTC-PD. Taking into account the overall gain of $41 \mathrm{~dB}$ provided by the THz amplifier cascade as well as the $2.5 \mathrm{~dB}$ loss in the THz probe, the overall antenna gain of the transmitter and the receiver antennalens combination in our experiment amounts to approximately $79 \mathrm{dBi}$. For a span of $d=1 \mathrm{~km}$, the FSPL would increase to about $142 \mathrm{~dB}$. At the same time, we may assume that the POH MZM can be improved to feature a lower $U_{\pi} L$ product of, e.g., $100 \mathrm{~V} \mu \mathrm{m}$, which is a factor of 2.4 smaller than the $U_{\pi} L$ product of $240 \mathrm{~V} \mu \mathrm{m}$ of the current device. Assuming the same modulator length as in the current experiment, the required $\mathrm{THz}$ drive power to obtain a similar BER performance as the one shown in Fig $3 \mathrm{~d}$ of the main paper would drop to approximately $-8 \mathrm{dBm}$. Using the same UTC-PD and THz amplifiers as in the current experiment, one would require an overall antenna gain of about $106 \mathrm{dBi}$, which can be realistically realized [21]. Additional improvements may be achieved by using further optimized EO materials, for which $U_{\pi} L$ products of the order of $35 \mathrm{~V} \mu \mathrm{m}$ can be attained, see Section V, contributing another $9 \mathrm{~dB}$ to the power budget. Moreover, co-packaging of THz amplifiers and POH MZM may help to eliminate the losses of the THz connections and the probe, and use of high-power THz UTC-PD [22], in combination with $\mathrm{THz}$ amplifier (e.g., MPA) at the transmitter, can permit higher transmitter powers of, e.g. $4 \mathrm{dBm}$, which would add at least another $15 \mathrm{~dB}$ to the power budget. Note also that, for high data rates, any improvement of the optical losses of the POH MZM contributes to a further increase in the loss margin. Taken together, these measures should allow to increase the transmission distance to $1 \mathrm{~km}$ or more, while still maintaining a reasonable loss margin for the wireless THz link.

Another unique feature of $\mathrm{POH}$ modulators is their compact footprint, which, when used in conventional transceivers, offers large communication bandwidth on a small chip area. Taking into account silicon photonic MMI couplers along with the POH phase shifter section, a T/O converter would occupy an on-chip area of approximately $40 \mu \mathrm{m} \times 10 \mu \mathrm{m}=400 \mu \mathrm{m}^{2}$ and features a $3 \mathrm{~dB}$ frequency response exceeding $0.36 \mathrm{THz}$, thus leading to a bandwidth-to-area ratio larger than $900 \mathrm{THz} / \mathrm{mm}^{2}$. Considering the aforementioned improvements with respect to the use of polymers with larger EO coefficients (a seven-fold increase in the

$n_{\mathrm{EO}}^{3} r_{33}$ ) and assuming that the power splitters can be designed using the plasmonic waveguides [3], the total area of the POH MZM can be brought down to approximately $5 \mu \mathrm{m} \times 2 \mu \mathrm{m}=10 \mu \mathrm{m}^{2}$, resulting in a bandwidth-to-area ratio as large as $36 \mathrm{PHz} / \mathrm{mm}^{2}$. In comparison, the state-of-the-art silicon depletion-type modulators [17] have a bandwidth-to-area ratio of, e.g., $3.2 \mathrm{THz} / \mathrm{mm}^{2}$ ( $3 \mathrm{~dB}$ frequency of $48 \mathrm{GHz}$ with a modulator length of $250 \mu \mathrm{m}$ and assuming a minimum width of $\left.60 \mu \mathrm{m}\right)$ which is at least a factor of 280 smaller than what could be achieved with the POH technology. Note that these numbers are merely estimations, which completely disregard the necessary electrical and optical feed lines. The coupling structures for the THz signals such as contact pads or $\mathrm{THz}$ antennae would dominate the device footprint and eventually limit integration density. In a general practice, MMIC chips are coupled to the rectangular waveguides terminations of bulk devices, for e.g., high-gain horn antenna, through radial-probe transitions [23]. Following a similar approach for T/O converters requires a considerable chip area of at least $0.4 \mathrm{~mm}^{2}$, corresponding to the dimensions of the rectangular waveguide. Alternatively, T/O converters could be integrated with on-chip planar antenna structures [24], [25] which have relaxed space requirements of less than $0.1 \mathrm{~mm}^{2}$ but come at a cost of smaller antenna gain and increased cross-talk. However, these coupling structures are still much larger than the footprint of the $\mathrm{POH}$ devices.

\section{References}

[1] Y. Shi, L. Yan, and A. E. Willner, 'High-speed electrooptic modulator characterization using optical spectrum analysis', $J$. Lightwave Technol., vol. 21, no. 10, pp. 2358-2367, 2003. 
[2] C. Koos et al., 'Silicon-organic hybrid (SOH) and plasmonic-organic hybrid (POH) integration', J. Lightwave Technol., vol. 34, no. 2, pp. 256-268, 2016.

[3] C. Haffner et al., 'All-plasmonic Mach-Zehnder modulator enabling optical high-speed communication at the microscale', Nat. Photon., vol. 9, no. 8, pp. 525-528, Jul. 2015.

[4] C. Hoessbacher et al., 'Plasmonic modulator with $>170 \mathrm{GHz}$ bandwidth demonstrated at $100 \mathrm{GBd}$ NRZ', Opt. Express, vol. 25, no. 3, pp. 1762-1768, 2017.

[5] W. Jin et al., 'Structure-function relationship exploration for enhanced thermal stability and electro-optic activity in monolithic organic NLO chromophores', J. Mater. Chem. C, vol. 4, no. 15, pp. 3119-3124, 2016.

[6] 'Operating Manual: Millimeter-Submillimeter Power Meter, VDi Erickson PM4', Virginia Diodes, 2010.

[7] A. Tessmann et al., 'High-gain submillimeter-wave mHEMT amplifier MMICs', IEEE MTT-S Int. Microw. Symp. Dig., pp. 53-56, 2010.

[8] R. Schmogrow et al., 'Error vector magnitude as a performance measure for advanced modulation formats', IEEE Photonics Technol. Lett., vol. 24, no. 1, pp. 61-63, 2012.

[9] R. Schmogrow et al., 'Erratum: Corrections to error vector magnitude as a performance measure for advanced modulation formats (IEEE Photonics Technology Letters (2012) 24:1 (61-63))', IEEE Photonics Technol. Lett., vol. 24, no. 23, p. 2198, 2012 .

[10] W. Freude et al., 'Quality metrics for optical signals: Eye diagram, Q-factor, OSNR, EVM and BER', in 14th International Conference on Transparent Optical Networks, 2012, p. Mo.B1.5 (invited).

[11] T. Pfau, S. Hoffmann, and R. Noe, 'Hardware-Efficient Coherent Digital Receiver Concept With Feedforward Carrier Recovery for M-QAM Constellations', J. Lightwave Technol., vol. 27, no. 8, pp. 989-999, 2009.

[12] C. Haffner et al., 'Harnessing nonlinearities near material absorption resonances for reducing losses in plasmonic modulators', Opt. Mater. Express, vol. 7, no. 7, pp. 2168-2181, 2017.

[13] C. Kieninger et al., 'Ultra-high electro-optic activity demonstrated in a silicon-organic hybrid (SOH) modulator', Optica, vol. 5, no. 6, pp. 739-748, Sep. 2018.

[14] W. Heni et al., 'Nonlinearities of organic electro-optic materials in nanoscale slots and implications for the optimum modulator design', vol. 25, no. 3, pp. 2627-2653, 2017.

[15] K. M. McPeak et al., 'Plasmonic Films Can Easily Be Better: Rules and Recipes', ACS Photonics, vol. 2, no. 3, pp. 326$333,2015$.

[16] R. Ding et al., 'High-speed silicon modulator with slow-wave electrodes and fully independent differential drive', $J$. Lightwave Technol., vol. 32, no. 12, pp. 2240-2247, 2014.

[17] S. S. Azadeh et al., 'Low V $\pi$ silicon photonics modulators with highly linear epitaxially grown phase shifters', Opt. Express, vol. 23 , no. 18 , p. $23526,2015$.

[18] M. Burla et al., '500 GHz plasmonic Mach-Zehnder modulator enabling sub-THz microwave photonics', Prepr. arXiv1901.00477, 2018.

[19] Y. Ding, H. Ou, and C. Peucheret, 'Ultrahigh-efficiency apodized grating coupler using fully etched photonic crystals', Opt. Lett., vol. 38, no. 15, p. 2732, 2013.

[20] D. Benedikovic et al., 'Subwavelength index engineered surface grating coupler with sub-decibel efficiency for 220-nm silicon-on-insulator waveguides', Opt. Express, vol. 23, no. 17, p. 22628, 2015.

[21] T. Nagatsuma, G. Ducournau, and C. C. Renaud, 'Advances in terahertz communications accelerated by photonics', Nat. Photon., vol. 10, no. 6, pp. 371-379, 2016.

[22] H. J. Song, K. Ajito, Y. Muramoto, A. Wakatsuki, T. Nagatsuma, and N. Kukutsu, 'Uni-travelling-carrier photodiode module generating $300 \mathrm{GHz}$ power greater than $1 \mathrm{~mW}$ ', IEEE Microw. Wirel. Components Lett., vol. 22, no. 7, pp. 363365, 2012.

[23] H. J. Song, 'Packages for terahertz electronics', Proc. IEEE, vol. 105, no. 6, pp. 1121-1138, 2017.

[24] Y. Salamin et al., 'Microwave plasmonic mixer in a transparent fibre-wireless link', Nat. Photon., vol. 12, no. 12, pp. 749753, 2018. 
[25] T. Harter et al., 'Silicon-plasmonic integrated circuits for terahertz signal generation and coherent detection', Nat. Photon., 2018. 